\title{
La diversité légitime : nouveau défi de l'intégration européenne
}

In: Revue française de science politique, 52e année, n5-6, 2002. pp. 609-639.

Article by an MPIfG researcher

Fritz W. Scharpf: La Diversité Légitime: Nouveau Défi de l'Intégration Européenne. In: Revue Française de Science Politique

52(5-6), 609-639 (2002). Presses de Sciences Po

The original publication is available at the publisher's web site: http://dx.doi.org/10.3406/rfsp.2002.403742

Citer ce document / Cite this document :

Scharpf Fritz W. La diversité légitime : nouveau défi de l'intégration européenne. In: Revue française de science politique, 52e année, n5-6, 2002. pp. 609-639.

doi : $10.3406 /$ rfsp.2002.403742

http://www.persee.fr/web/revues/home/prescript/article/rfsp_0035-2950_2002_num_52_5_403742 


\section{Résumé}

Discuter apropos d'une constitution européenne nécessite de se confronter à d'importants défis politiques qui ne se rencontrent pas réellement dans le cadre institutionnel actuel de l'Union européenne. Le présent article présente tout d'abord trois modes de gouvernement communau-taire distincts : les négociations intergouvernementales, la centralisation supranationale et les décisions conjointes. Dans ces trois modes de gouvernement, l'action européenne appliquée et légitime dépend d'un haut degré de consensus entre les membres des gouvernements des États. L'article examine ensuite les conditions des réponses européennes aux trois nouveaux défis politiques : l'élargissement à I'Est, la politique communautaire extérieure et de sécurité ainsi que la protection des États providence européens face à l'impact de l'intégration économique et de la libéralisation du marché. Dans chaque cas, on peut voir que les conflits d'intérêt signi-ficatifs entre les membres des gouvernements empêchent toute action dans le cadre institu-tionnel actuel. Prétendant que ces conflits ne peuvent pas être résolus par une évolution vers des institutions à régime majoritaire, l'article conclut en proposant différentes réponses poli-tiques qui permettraient l'action communautaire dans le cadre d'une diversité légitime.

\section{Abstract}

Legitimate diversity : the new challenge of european integration

Discussions about a European constitution ought to focus on substantive policy challenges that cannot be met effectively within the present institutional framework of the European Union. The paper begins by discussing the characteristics of three distinct modes of European policy making intergovernmental agreement, supranational centralization and joint-decision making. In all three modes, effective and legitimate European action depends on high levels of substantive consensus among member state governments. The paper goes on to examine the requirements of effective European responses to three novel policy challenges - Eastern enlargement, common foreign and security policy and the protection of European welfare states against the impact of economic integration and liberalization. In each case, it can be shown that politically salient conflicts of interest among member governments will prevent effective action within the present institutional framework. Arguing that these conflicts could not be legitimately settled by a move toward majoritarian institutions, the paper concludes with a discussion of dif-ferentiated policy responses that would allow European action in the face of legitimate diversity. 


\title{
LA DIVERSITÉ LÉGITIME : NOUVEAU DÉFI DE L'INTÉGRATION EUROPÉENNE
}

\author{
FRITZ W. SCHARPF
}

\section{L'IMPASSE DES DÉBATS CONSTITUTIONNELS ACTUELS}

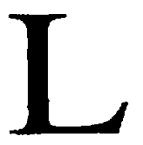

e débat public sur une Constitution européenne, alimenté par les frustrations faisant suite aux maigres résultats du sommet de Nice, semble devoir aboutir

à une impasse avant même d'avoir commencé. Cela était peut-être inévitable après que Joschka Fischer ', dans son discours à l'université Humboldt, eut demandé rien moins qu'un accord sur le fait d'inscrire la création d'un État fédéral démocratique comme finalité institutionnelle de l'Union. Une fois le débat posé en ces termes, la suite des événements était prévisible. On pouvait s'attendre à un soutien de la part du Parlement européen et, avec quelques réserves, des pays du Bénélux et de l'Italie - soutien auquel Gerhard Schröder, s'adressant aux sociaux-démocrates et en particulier aux Länder, ajouta un ceterum censeo concernant les limitations constitutionnelles des pouvoirs de l'Union ${ }^{2}$. Il était tout aussi prévisible que le Royaume-Uni et la Scandinavie s'y opposent farouchement, tandis qu'on tardait à savoir si la France allait se ranger du côté des défenseurs de la souveraineté nationale ou bien finir par conclure que son aspiration de longue date à créer une «puissance mondiale » européenne devait s'appuyer sur des institutions fédérales. En fait, la réponse du président Jacques Chirac à Berlin ${ }^{3}$ éluda la question du choix entre les finalités institutionnelles, pour réaffirmer la priorité accordée, dans une perspective pragmatique, à une coopération renforcée entre les pays du « groupe pionnier » désireux d'avancer plus rapidement vers un objectif non précisé d'intégration européenne. Un an après, cependant, le Premier ministre, Lionel Jospin, renversa l'ordre des priorités. Un accord sur les buts communs poursuivis par l'Europe et sur des réalisations concrètes devait précéder et guider l'élaboration de projets institutionnels. Il ne laissa toutefois planer aucun doute sur le fait que la vision allemande d'un État fédéral européen n'était pas acceptable et qu'une Union comprise comme une « fédération d'États nations », tout en nécessitant des réformes institutionnelles afin de renforcer son efficacité et sa fiabilité, devrait continuer à s'appuyer sur le triangle institutionnel formé par le Conseil, la Commission et le Parlement.

Il y a de bonnes raisons, d'ordre à la fois pragmatique et théorique, de suivre l'ordre des priorités fixé par Jospin. Un débat sur les finalités constitutionnelles dans

1. Joschka Fischer, « From Confederacy to Federation : Thoughts on the Finality of European Integration », dans Christian Joerges, Yves Mény, J. H. H. Weiler (eds), What Kind of Constitution for What Kind of Polity? Responses to Joschka Fischer, Florence, European University Institute, $19-30<\mathrm{http}: / / \mathrm{ww}$ w.iue.it/RCS/symposium/>.

2. Gerhard Schroeder, « SPD Leitantrag "Verantwortung für Europa" », projet du 29 avril $2001<$ http://www.europa-digital.de/text/aktuell/dossier/reden/>.

3. Discours de Jacques Chirac devant le Bundestag, 27 juin $2000<$ http://www.mondediplomatique.fr/cahier/europe/discourschirac>. 
l'abstrait est forcé d'aboutir à des malentendus insurmontables : ce que les Allemands mettent sous le terme de «fédéralisme »n'est pas ce que comprennent les Français - ou bien ce que les Britanniques ont l'air d'entendre ${ }^{1}$. Il en irait de même avec d'autres concepts clés, qui tous voient leur signification définie au sein des traditions constitutionnelles spécifiques à chaque État membre - et dont les différences peuvent sans doute être perçues par des spécialistes de politique comparée, mais pas par un plus vaste public. De plus, la recommandation faite par Jospin a l'histoire de son côté. À chaque fois - de la création de la CEE au Marché commun et à l'union monétaire - les innovations institutionnelles les plus importantes furent précédées, facilitées et justifiées par un accord sur les objectifs politiques concrets pour l'accomplissement desquels ces innovations étaient jugées nécessaires et acceptables.

Cette pratique historique trouve sa justification théorique dans le fait qu'il n'y a qu'une seule forme institutionnelle d'intégration européenne qui puisse être débattue et évaluée sans qu'il soit fait référence à des objectifs ou des problèmes politiques concrets et précis, à savoir la constitution d'un État démocratique aux compétences universelles. Si cette forme d'État était accessible et désirable - et elle compte parmi ses partisans des spécialistes de philosophie politique, des membres du Parlement européen et peut-être Joschka Fischer lorsqu'il s'exprime à titre privé il resterait encore à débattre de ses avantages relatifs et de l'équilibre à trouver entre différentes formes d'État possibles : État fédéral ou unitaire, présidentiel ou parlementaire, à régime majoritaire ou consensuel ${ }^{2}$, mais sans qu'il soit absolument nécessaire de débattre des projets et des objectifs. Ces derniers pourraient et devraient être confiés aux futurs processus démocratiques, tels que définis par la nouvelle constitution.

On peut raisonnablement affirmer, cependant, même à ce stade initial des débats constitutionnels, que la plupart des acteurs représentant « les peuples d'Europe » dans la série en cours de réformes institutionnelles ne souhaitent pas, ou tout du moins pas encore, créer un État européen à compétences universelles. Dans ces circonstances, le débat doit tourner autour de la réforme des institutions actuelles de l'Union, avec pour conséquences que celui-ci sera gouverné, implicitement ou explicitement, par la logique pragmatique recommandée par Lionel Jospin, et mise en ceuvre naguère aussi bien par Jacques Delors que par Jean Monnet. Les institutions actuelles ont permis et rendu légitime la création du Marché unique et de l'union monétaire ; les prochaines réformes doivent être reconnues comme nécessaires et légitimes pour faire face à de nouveaux défis. Ainsi, le débat constitutionnel devrait porter sur les prochains objectifs que nous souhaitons nous fixer - ou, ce qui est plus urgent encore, sur les problèmes auxquels nous devons dès à présent faire face - et dire pourquoi les institutions européennes dans leur forme actuelle ne sont pas adaptées à ces aspirations. C'est seulement sur cette base que l'on peut espérer un accord sur les réformes institutionnelles qui puisse se justifier, alors même qu'on se trouve à un tournant historique de l'intégration européenne.

1. Au cours d'une conférence franco-allemande récente, Michel Albert, certainement l'un des amis les mieux informés que l'Allemagne ait en France, a loué la BCE comme étant une institution modèle pour une future Europe fédérale. Pourtant, en Allemagne, chacun considèrerait une Bundesbank dotée de pouvoirs similaires comme un exemple de gouvernance extrêmement centralisée, très éloigné du modèle allemand de fédéralisme coopératif.

2. Arend Lijphart, Patterns of Democracy: Government Forms and Performance in Thirty-Six Countries, New Haven, Yale University Press, 1999. 
Puisqu' un accord sur les objectifs est moins probable qu'un accord sur l'existence de problèmes manifestes, mon analyse portera essentiellement sur ces derniers. J'essaierai de montrer que l'Union européenne est bel et bien confrontée à d'importantes questions dans des domaines de la politique générale où il est difficile, voire impossible de trouver des solutions dans le cadre institutionnel actuel. Les plus importantes de ces questions sont les suivantes :

La question de la politique étrangère et de sécurité commune, qui est devenue cruciale dans les Balkans et après le 11 septembre 2001 ;

La question relative à l'élargissement de l'Union à l'Est ;

La question qui se pose aux États membres après la mise en place réussie du marché intérieur et de l'union monétaire.

Mais, avant d'examiner ces questions et la remise en cause des institutions européennes qu'elles impliquent, il me faut donner un bref aperçu du fonctionnement actuel de ces institutions. Afin de simplifier les choses, je ne partirai pas des structures institutionnelles en tant que telles, mais de trois modes ' ${ }^{1}$ abstraits de prises de décisions que je désignerai sous le nom de (1) «négociations intergouvernementales», (2) «prise de décision commune ou conjointe $»$ et (3) «centralisation supranationale $»^{2}$. Il me sera ensuite possible d'examiner les problèmes sus-mentionnés à la lumière des forces et des faiblesses inhérentes à ces trois modes de prise de décision.

\section{LA PLURALITÉ DES MODES DE GOUVERNEMENT EUROPÉEN}

Comme pour les systèmes politiques des États nations ${ }^{3}$, l'Union européenne fonctionne différemment selon les domaines d'application de sa politique générale. Toutefois, les institutions qui sont à la tête de l'Europe étant créées par les États membres, le premier mode de prise de décision, quels que soient les domaines de politique générale concernés, doit être intergouvernemental. Ce sont les gouvernements respectifs des États membres qui décident que certains choix politiques - qui sinon seraient effectués de manière autonome dans le cadre du système politique de chaque État membre - devraient l'être au niveau européen. Au cours du même processus, les gouvernements doivent décider selon quelles modalités ces choix européens devront être effectués. Les gouvernements peuvent opter pour des «accords intergouvernementaux » ou bien pour un mode de prise de décision commun, impliquant la Commission, le Conseil et le Parlement, ou encore, décider de donner tout pouvoir à la Commission, à la Cour de justice ou à la Banque centrale pour prendre des décisions

1. J'utilise le terme de «mode » pour caractériser la combinaison de règles définissant les droits de participation et les règles de décision applicables.

2. Fritz W. Scharpf, « Notes Toward a Theory of Multilevel Governing in Europe », Scandinavian Political Studies, 24, 2001, p. 2-26.

3. En Allemagne, par exemple, le mode caractéristique de gouvernement dans de nombreux secteurs politiques est une forme de prise de décision commune associant le gouvernement fédéral et sa majorité parlementaire dans des négociations avec les Länder; dans d'autres secteurs de la politique, cependant, les Länder n'ont aucun rôle dans la prise de décision et le gouvernement est en revanche impliqué dans des négociations «néo-corporatistes » avec des associations de premier plan; dans d'autres secteurs de la politique encore, le mode de gouvernement est carrément majoritaire ; et, étant donné le rôle capital joué par la Cour constitutionnelle et la Bundesbank, des décisions politiques importantes sont aussi prises de manière centralisée. 
contraignantes, sans davantage de concertation avec les gouvernements membres ${ }^{1}$. Ces modes de prise de décision diffèrent dans leur aptitude à effectuer des choix efficaces face à des conflits d'intérêts entre les États membres ; et en conférant un pouvoir à des acteurs différents, ils infléchissent les résultats probables des politiques mises en œuvre ${ }^{2}$. Ces mécanismes décisionnels diffèrent aussi selon l'éventail de décisions qu'ils sont susceptibles de mettre en œuvre légitimement ${ }^{3}$.

\section{CENTRALISATION SUPRANATIONALE}

Dans le cadre européen, le mode de prise de décision centralisé est un processus en deux ou trois étapes. Fondamentalement, il doit y avoir accord intergouvernemental sur l'européanisation du domaine d'application. Cet accord peut aussi porter sur un choix politique de base dont le détail et l'application sont laissés à une institution supranationale qui a le droit d'exercer son pouvoir discrétionnaire sans la participation des gouvernements nationaux. L'exemple le plus clair d'un mode de décision en deux étapes est fourni par le rôle décisif joué par la Banque centrale européenne dans la politique monétaire européenne. Le mandat qu'elle a reçu de « maintenir la stabilité des prix » (art. 105, traité de la Communauté européenne) fut défini par les négociations du Traité ; et dans l'application de ce mandat, la Banque européenne est mieux protégée de l'influence des gouvernements des États membres de l'union monétaire que n'importe quelle autre banque centrale nationale, y compris la Bundesbank.

On retrouve la même procédure en deux temps dans tous les domaines politiques où les traités incluent des interdictions et des obligations - directement applicables adressées aux États membres, ou des droits concernant les individus et les entreprises. Dans ce cas, le pouvoir conféré à la Commission d'engager des poursuites pour violation des traités contre certains États membres et celui conféré à la Cour de justice européenne d'émettre des interprétations formellement contraignantes et exécutoires de ces obligations conventionnelles ne peuvent être remis en cause que par des amendements du texte des traités, adoptés et ratifiés à l'unanimité. Ainsi, en pratique, cette

1. Une théorie qui voudrait expliquer (de manière rationnelle) ces choix entre différents modes de gouvernement devrait considérer simultanément la pression exercée par les problèmes qui ne peuvent pas être résolus au niveau purement national et celle exercée par les problèmes auxquels les gouvernements nationaux auraient à faire face si les décisions européennes devaient remettre en cause d'importants intérêts nationaux ou des préférences politiquement sensibles auprès des électeurs. Ces problèmes pourraient être représentés par trois constellations de base de la théorie des jeux : le Dilemme du prisonnier, la Bataille des sexes et un jeu où les intérêts communs sont dominés par les conflits concernant le choix d'une solution.

2. Adrienne Héritier, « Market Integration and Social Cohesion: The Politics of Public Services in European Regulation ", Journal of European Public Policy, 8, 2001, p. 825-852. Même si Adrienne Héritier ne fait explicitement référence qu'aux règles de décision, les distinctions qu'elle établit sont parallèles aux trois modes de prise de décision dont il est ici question. Se concentrant sur la régulation dans le secteur des services publics, elle montre comment le choix entre ces trois modes décisionnaires affecte le poids relatif donné aux partisans du libéralisme ou de la cohésion sociale.

3. Ibid. Adrienne Hériter montre aussi qu'alors même que les domaines de la politique sont associés de manière institutionnelle à un mode donné de prise de décision (c'est-à-dire, concurrence $\rightarrow$ mode supranational-hiérarchique, marché intérieur $\rightarrow$ mode commun de prise de décision(s)) des initiatives politiques spécifiques peuvent très bien être lancées sous un mode institutionnel ou un autre - ce qui implique que le choix du mode puisse devenir luimême un enjeu stratégique. 


\section{Un nouveau défi de l'intégration européenne}

possibilité d'interprétation des traités permet une prise de décision supranationale et centralisée dans les domaines où les dispositions conventionnelles sont directement applicables.

À quelques rares exceptions près (l'une d'elles étant l'interdiction de la discrimination sexuelle dans les relations de travail), ces conditions s'appliquent dans les domaines politiques visant à favoriser l'intégration économique et la libéralisation du marché. En l'occurrence, une fois d'accord sur les engagements de base, la constellation d'intérêts peut en effet être interprétée comme un dilemme du prisonnier symétrique : tous les États membres ont intérêt à ce que les engagements soient tenus, mais tous sont aussi tentés de recourir à des pratiques protectionnistes. Au cas où un tel risque est anticipé, la délégation du pouvoir exécutoire à des acteurs supranationaux, telles que la Commission et la Cour de justice, est légitimée du fait même que les États membres y trouvent leur propre intérêt ' . Si ce cadre conceptuel est admis, il est alors totalement acceptable de voir des décisions individuelles entrer en conflit avec les intérêts à court terme des gouvernements nationaux ${ }^{2}$. Cela implique néanmoins que l'évolution, échappant au contrôle politique, du droit de la concurrence européen conduirait à des interprétations des engagements conventionnels par la Cour de justice qui pourraient aller (comme ce fut souvent le cas) au-delà des intentions premières des gouvernements à l'origine des traités 3 .

Dans une moindre mesure, le même type de pouvoir décisionnel existe dans le cadre d'un processus en trois étapes, là où un accord intergouvernemental dans les traités a créé une compétence européenne, tout en laissant la définition des choix politiques concrets aux directives et aux régulations adoptées au cours de processus décisionnels communs intéressant la Commission, le Conseil et, de plus en plus souvent, le Parlement. Dans l'exécution de ces règles plus spécifiques du droit européen « dérivé », la latitude d'interprétation laissée à la Commission et à la Cour est plus faible ; mais, comme de telles règles ne peuvent être modifiées qu'avec l'accord d'au moins la majorité qualifiée des votes au Conseil, il reste une possibilité pour que s'exerce, dans ce cas aussi, une forme de prise de décision supranationale et centralisée.

Ce sont ces pouvoirs exécutoires centralisés exercés par la Commission et la Cour qui expliquent les progrès de l'intégration économique dans les périodes de stagnation politique des années 1970 et 1980 ; ils expliquent aussi le progrès et la radicalisation rapides des politiques de libéralisation du marché, une fois les engagements politiques de base entérinés par la signature de l'Acte unique européen de 1986. De plus, ces pouvoirs exécutoires peuvent aussi être employés à des fins stratégiques par la Commission afin d'inciter des gouvernements réticents à accepter de nouvelles lois susceptibles, encore une fois, de faire avancer la libéralisation économique ${ }^{4}$. Ainsi, certains gouvernements attachés à la défense de leurs services et de leurs infrastructures publics, en contravention des lois sur la concurrence, n'ont jusqu'à maintenant pas

1. Andrew Moravcsik, The Choice for Europe. Social Purpose and State Power from Messina to Maastricht, Ithaca, Cornell University Press, 1998.

2. Anne-Marie Burley, «Walter Matti, Europe Before the Court: Political Theory of Legal Integration », International Organization, 47, 1993, p. 41-76.

3. Fritz W. Scharpf, Governing in Europe : Effective and Democratic ?, Oxford, Oxford University Press, 1999.

4. Susanne K. Schmidt, «Commission Activism: Subsuming Telecommunications and Electricity under European Competition Law », Journal of European Public Policy, 5, 1998, p. $169-184$. 
réussi à obtenir de révisions des traités qui imposeraient des limitations légales à la libéralisation du marché.

\section{PRISE DE DÉCISION COMMUNE}

Le mode normal de prise de décision dans le « premier pilier » de la Communauté européenne a les caractéristiques d'un processus d'accord commun impliquant les acteurs supranationaux ainsi que les gouvernements nationaux. Il peut prendre la forme soit de « directives» qui doivent être transposées dans le cadre des législations nationales suivant les processus législatifs des États membres, soit de « règlements » applicables directement. Directives et règlements doivent être adoptés, à l'initiative de la Commission, par le Conseil des ministres qui a recours de plus en plus fréquemment au vote à la majorité qualifiée, et par le Parlement européen dont le rôle a été renforcé à travers l'utilisation accrue des procédures de co-décision ${ }^{1}$. Lors de la préparation de ses initiatives, la Commission consulte un large éventail de groupements d'intérêt, d'entreprises, d'organisations non-gouvernementales et de comités d'experts. De même, lorsqu'il met sur pied sa position commune, le Conseil s'appuie sur le Comité de représentants permanents (COREPER) et sur un grand nombre de commissions spécialisées représentant les gouvernements des États membres. Le rôle du Parlement ayant été renforcé, les commissions spéciales du Parlement européen et les négociations entre celles-ci et les commissions du Conseil ont aussi acquis une importance accrue. De plus, si les directives doivent être appliquées au moyen de régulations plus fines, la charge en revient généralement à la Commission, qui opère alors selon des procédures de «comitologie » faisant appel une fois encore à des fonctionnaires et à des experts nommés par les gouvernements membres.

Prises ensemble, ces procédures institutionnelles ménagent un si grand nombre de possibilités de veto et de points d'accès pour des groupements d'intérêt, qu'il est peu probable que les politiques mises en œuvre par les procédures de prise de décision commune remettent en cause des acquis politiquement sensibles pour les États membres ou qui sont défendus par des lobbies bien organisés. Dans le même temps cependant, le rôle central de la Commission et la présence de représentants nationaux dans le COREPER et au sein des comités de Comitologie permettent généralement d'éviter que les positions initialement conflictuelles soient prises au pied de la lettre, et laisse ainsi la voie libre à l'exploration de solutions créatives et équitables ou à l'établissement de compromis acceptables par toutes les parties. Par conséquent, on parvient à des accords plus souvent que ne le laisserait penser une analyse statique des intérêts nationaux et l'on peut estimer que les solutions dégagées trouvent généralement une légitimité fondée sur un large consensus ${ }^{2}$.

Mais, en même temps, ces négociations multipartites sont non seulement complexes mais aussi peu transparentes - situation qui est source de nombreuses critiques eu égard à la violation des normes démocratiques. Dans une optique critique sûrement plus pertinente, il a été relevé que le mode de prise de décision commun, comme tous

1. Adrienne Héritier, art. cité. Elle a montré comment les pouvoirs dévolus au Parlement ont renforcé le rôle des principes de cohésion sociale dans la régulation de la libéralisation des services publics.

2. Volker Eichener, Das Entscheidungsystem der Europäische Union : institutionelle Analyse und demokratietheoretische Bewertung, Opladen, Leske \& Budrich, 2000. 
les systèmes à veto multiple ', a tendance à favoriser le statu quo aux dépens des choix politiques qui ne pourraient être mis en œuvre qu'au prix de changements effectifs. De plus, les processus fondés sur une recherche du consensus sont lents et, quand ils aboutissent, la législation qui s'ensuit repose souvent sur le plus petit dénominateur commun ou est encombrée de trop nombreux détails ${ }^{2}$. En d'autres termes, la performance du mode de prise de décision conjoint est limité dans les domaines politiques sensibles auprès de l'électorat des pays membres et, en général, l'efficacité des politiques qui peuvent être adoptées de cette manière laisse beaucoup à désirer.

\section{ACCORD INTERGOUVERNEMENTAL}

L'accord intergouvernemental n'est pas réservé aux décisions fondatrices permettant l'européanisation des politiques publiques dans des domaines jusqu'ici laissés au contrôle autonome de chaque pays, soit par des révisions explicites des traités, soit par un accord unanime au sein du Conseil sous la clause « nécessaire et approprié » de l'art. 308 (ex 235) du TCE. Ce type d'accord s'applique aussi dans des domaines où les gouvernements ont reconnu la nécessité d'une action au niveau européen mais où, du moins pour certains, le coût (économique ou politique) de décisions allant contre leurs préférences serait si élevé qu'ils ne peuvent pas accepter un vote à la majorité qualifiée au Conseil. Ainsi, la règle de l'unanimité a-t-elle été maintenue dans les domaines de l'harmonisation fiscale, des choix budgétaires et de certains secteurs de la politique sociale. Si leurs réserves sont encore plus fortes, les gouvernements souhaiteront éviter d'être mis dans l'embarras par les initiatives législatives de la Commission, ou par l'obligation de négocier des compromis avec le Parlement, et ils chercheront donc à priver la Commission et la Cour de justice de leurs prérogatives en termes d'interprétation et d'application des règlements. Ce sont ces conditions qui ont caractérisé les prises de décision intergouvernementales dans les deuxième et troisième piliers du traité de l'Union européenne - «Politique étrangère et sécurité commune », « Justice-Affaires intérieures »-bien que certaines compétences qui leur étaient jusqu'à présent rattachées - visas, droit d'asile, immigration - soient sur le point d'être incluses aux règles gouvernant le premier pilier (articles 61-69 TCE). Au cours des dernières années, enfin, le Conseil européen a de plus en plus souvent contourné les prérogatives de la Commission dans l'initiative des lois, en définissant certains éléments du calendrier politique européen au cours de ses réunions au sommet - éléments qui doivent alors être mis au point par la voie législative ou par le biais d'arrangements intergouvernementaux ad hoc.

Dans tous ces cas de figure, les gouvernements nationaux ont un droit de veto, qu'ils peuvent exercer dans la défense obstinée d'intérêts nationaux (ou économiques) étroits et à court terme, ou bien pour défendre des intérêts européens communs ou

1. George Tsebelis, «Decision-Making in Political Systems : Veto Players in Presidentialism, Parliamentarism, Multicameralism, and Multipartyism », British Journal of Political Science, 25, 1995, p. 289-326.

2. Les deux cas de figure sont tout à fait possibles dans des systèmes à veto multiple : les pays à faible réglementation ne trouvant aucun intérêt dans les solutions européennes communes peuvent opposer leur veto à toutes les règles imposant une meilleure protection. En revanche, les pays à forte réglementation qui ont intérêt à une solution commune peuvent toujours menacer d'utiliser leur droit de veto pour s'assurer que leur propre système de règles soit inclus dans cette solution commune. 
encore dans l'espoir de tirer des bénéfices à plus long terme d'une meilleure coopération et coordination politiques. Quoi qu'il en soit, les résultats de négociations menées sur le mode intergouvernemental ont en général une plus grande visibilité que les politiques européennes adoptées selon les modes de prise de décision supranationaux ou communs, ce qui signifie par ailleurs qu'ils tombent plus facilement sous le regard critique des partis d'opposition et des média, et que les gouvernements doivent avoir la capacité et la volonté de défendre publiquement leur soutien à ces politiques communes en mettant en avant les avantages que pourraient en tirer leurs électeurs. Par conséquent, ces accords intergouvernementaux s'appuient sur la légitimité des gouvernements qui les concluent, mais leur efficacité dans la résolution des problèmes est limitée du fait même que les solutions apportées ne doivent pas aller contre les intérêts vitaux de l'électorat majoritaire.

\section{NOUVEAUX DÉFIS POLITIQUES}

Nos institutions européennes, il faut l'admettre, ont permis d'atteindre un degré d'unification des marchés, d'interaction économique et de mobilité interne qui dépasse largement les aspirations initiales des gouvernements lors de la signature du traité de Rome. Elles ont du même coup permis à l'Europe de l'Ouest de jouir d'une période de paix sans précédent. L'intégration européenne, qui a fait tomber les barrières économiques entre les États, a aussi réduit la signification politique des frontières nationales à un point tel qu'une guerre entre les membres de l'Union est devenue impensable. Pour aboutir à ces résultats, l'intégration européenne s'est appuyée sur des modes de gouvernement qui, quoique pas toujours très efficaces, étaient appropriés aux tâches à accomplir et dont la légitimité - bien qu'ils ne soient pas « démocratiques » au sens des démocraties constitutionnelles - $s$ 'appuie sur des arguments convaincants eu égard aux fonctions effectivement exercées.

Cependant, il est aussi clairement apparu que l'efficacité et la légitimité de ces modes de gouvernement sont limitées et qu'on ne peut pas, qu'on ne doit pas s'attendre à ce que, dans leur forme actuelle, ils puissent répondre à toutes sortes de problèmes nouveaux. Donc, si l'on veut que le débat constitutionnel actuel ait quelque portée pratique, il faut qu'il prenne en compte ces limites et donc la question de savoir si, dans leur forme actuelle, les institutions européennes sont susceptibles de résoudre les nouveaux défis qui attendent l'Union et les États membres.

\section{FORCES DE SÉCURITÉ COMMUNES (CFSP ET CESDP)}

Le défi le plus grave que doit relever l'Union est aussi celui dont je connais le moins bien les issues possibles. Dans les décennies qui ont suivi la guerre, les deux problèmes auxquels était confrontée la sécurité européenne, c'est-à-dire « maintenir les Russes à distance et s'assurer d'une Allemagne faible », furent résolus par l'alliance de l'OTAN sous la férule hégémonique des États-Unis. La fin de la Guerre froide vit disparaître la menace soviétique, tandis que le « danger » allemand - s'il avait jamais existé - s'était dissous au cours du processus d'intégration européen. Mais, avec l'effondrement du communisme, les conflits interethniques et religieux resurgirent, non seulement entre les anciennes républiques de l'Union soviétique, 
mais aussi plus près de l'UE, en Yougoslavie et chez ses voisins des Balkans. Bien que ces derniers ne constituent pas une menace militaire immédiate, il était clair que les États membres de l'UE seraient moralement et pratiquement affectés si des violences et un génocide avaient lieu à « leur porte », entrainnant l'arrivée massive de réfugiés et des conflits parmi leurs populations immigrées. Il ne fait aucun doute que le maintien et le rétablissement de la paix et de l'ordre dans les Balkans représentaient une urgence pour les États membres de l'UE.

Bien que l'OTAN ait fini par s'engager en Bosnie et au Kosovo, la réticence de la puissance américaine à s'impliquer dans des régions du monde où sa sécurité et ses intérêts économiques ne sont pas en jeu est devenue évidente. Mais, dans le même temps, les interventions militaires dans les Balkans ont révélé l'incapacité des pays européens, en dépit de leurs tentatives répétées pour coordonner leurs politiques étrangères, à trouver un accord sur des stratégies communes qui leur auraient permis d'intervenir ensemble avant que les violences ne s'exacerbent. Mais le plus important, c'est que les Européens se sont aperçus à cette occasion qu'ils manquaient, individuellement aussi bien que conjointement, des outils logistiques et de reconnaissance, des forces d'intervention entraînées et des armements spécialisés qui leur auraient permis d'engager leurs propres missions de maintien de la paix si les États-Unis n'avaient pas accepté de prendre la tête des opérations et d'en supporter le fardeau.

Depuis, les pays européens ont renforcé les institutions intergouvernementales leur permettant de coordonner leur politique étrangère et de sécurité commune ' et ont nommé son Haut représentant : Javier Solana. De plus, ils sont en train de développer leur potentiel militaire afin d'agir de manière autonome et de supporter la comparaison dans leur coopération avec les États-Unis. Ainsi, à Helsinski, ils se sont mis d'accord pour créer l'infrastructure institutionnelle d'une Politique européenne de sécurité et de défense commune (PESDC) qui inclut aussi un engagement pour tous les États membres (à l'exception du Danemark) à participer à une Force de réaction rapide européenne (FRRE). Au cours de ce processus, les gouvernements semblent s'être considérablement rapprochés dans leurs orientations, apparemment grâce à des échanges « trans-gouvernementaux » fréquents et intenses, impliquant les personnels militaires et diplomatiques et les élites des gouvernements concernés ${ }^{2}$.

Néanmoins, comme l'ont montré l'après-11 septembre et l'intervention en Afghanistan, les États-Unis continuent d'agir en fonction de leurs intérêts propres ; les réactions militaires et diplomatiques de leurs alliés européens sont, elles, définies au niveau national et au cours d'accords de coordination bilatéraux. Ainsi, les forces européennes ne constituent pas encore un facteur déterminant au plan international. De plus, la création de la Force de réaction rapide a été retardée par des restrictions budgétaires et par la difficulté à coordonner les diverses contributions nationales pour former une puissance militaire efficace. Dans le même temps, la question militaire étant souvent très sensible au niveau politique national, il est difficile de trouver un accord sur des stratégies communes, en particulier, dans les pays traditionnellement «neutres » et dans les pays où une large portion de la population soutient des positions

1. Stanley Hoffmann, «Towards a Common European Foreign and Security Policy ? », Journal of Common Market Studies, 38, 2000, p. 189-198.

2. Jolyon Howorth, «European Integration and Defence: The Ultimate Challenge ? », Paris, WEU-ISS, Chaillot paper, 43, 2000 ; Jolyon Howorth, « European Defence and the Changing Politics of the European Union: Hanging Together or Hanging Separatly ? », Journal of Common Market Studies, 39, 20001, p. 765-790. 
pacifistes. De toute façon, l'envoi de troupes est si étroitement associé à l'idée de souveraineté nationale et de démocratie que toute action militaire coordonnée reste suspendue à de longs débats nationaux.

En bref, même si la coordination entre les États membres s'est améliorée, la PESC et la PESDC sont toujours dépendantes du mode intergouvernemental de prise de décision qui, en l'absence d'un leadership américain vigoureux, continuera d'empêcher des réactions européennes rapides qui, au cours de la dernière décennie, auraient pu prévenir l'escalade de la violence et le recours tardif à une intervention massive. En même temps, étant donné la diversité des positions internationales et militaires de ses membres, il semble peu probable que l'UE dans son ensemble puisse faire passer la PESC/PESDC au mode de prise de décision commun, où les décisions nationales seraient prises par la Commission et par un vote à la majorité qualifiée au sein du Conseil des ministres de la défense (qui reste à créer). Inutile de dire qu'une solution supranationale et centralisée qui - par analogie avec la création de l'Union monétaire européenne - éliminerait tout contrôle des gouvernements nationaux et des parlements nationaux sur le déploiement des Forces de réaction rapide, paraît totalement hors de propos.

\section{ÉLARGISSEMENT À L'EST}

Le passage de l'UE de quinze à peut-être vingt-sept États membres au cours de la prochaine décennie constitue un sérieux défi. Les problèmes les plus conséquents, touchant aux règles de vote au Conseil et à la taille de la Commission, ont été débattus au sommet de Nice de manière peu convaincante. En fait, il a été démontré que, comparé aux règles actuelles du vote à la majorité qualifiée, le traité de Nice augmentera encore le seuil nécessaire pour atteindre une majorité décisive au sein du Conseil élargi ? Mais, le plus important, c'est le spectaculaire accroissement de l'hétérogénéité aux plans économique, social, culturel et institutionnel qu'induira l'élargissement à l'Est. Cela aura un effet sur la capacité de l'UE à adopter de nouvelles politiques au travers des modes de prise de décision intergouvernementaux et communs - problème sur lequel je reviendrai dans la section suivante. Mais cela aura aussi des conséquences sur les politiques existantes.

Comme je le faisais remarquer plus haut, l'application et l'exécution des lois de l'UE est confiée, via un processus de décision supranational et centralisé, à la Commission et à la Cour de justice (et aux tribunaux des États membres lorsqu'ils appliquent les décisions de la Cour de justice européenne dans leurs procédures légales ordinaires). Même si le pouvoir d'interpréter la loi a tendance à se transformer en prise de décision politique, la Commission et la Cour de justice restent normalement dans le cadre défini conjointement par les gouvernements qui ont participé à l'adoption des règles européennes, ce qui indique par ailleurs que les lois européennes et leur interprétation reflètent en gros les intérêts communs des pays qui étaient membres au moment de leur adoption. Comme l'expansion considérable de l'acquis communautaire au travers de l'Acte unique européen ne s'est effectuée qu' après l'élargissement au Sud et comme les derniers pays entrés dans l'Union - Finlande, Suède et Autriche -

1. George Tsebelis, Xenophon Yataganas, «Veto Players and Decision-Making in the EU after Nice: Legislative Gridlock and Bureaucratic/Judicial Discretion », Département de science politique, UCLA, 2001. 
sont très proches des premiers États membres, la mise en application de cet acquis n'a pas, jusqu'à présent, été considérée comme particulièrement problématique. Mais cela est appelé à changer avec l'élargissement de l'Union aux pays d'Europe centrale et orientale, dont les gouvernements n'ont pas participé à l'élaboration des très nombreux règlements européens et dont la situation et les intérêts sont fort différents de ceux qui prévalent dans les pays qui ont modelé l'Europe pendant plus de quarante ans '.

L'expérience de l'unification allemande donne une idée des problèmes à venir. En effet, l'application brutale des lois et des pratiques ouest-allemandes à l'ex-Allemagne de l'Est a entrâ̂né, après le démantèlement de ses industries, un chômage de masse, une désintégration sociale et politique qui persistent en dépit des transferts financiers s'élevant annuellement à cinq ou six pour cent du PIB ouest-allemand. Il est vrai que, d'ici à leur entrée dans l'UE, les pays d'Europe centrale et orientale auront eu une plus longue période d'adaptation au capitalisme et à la démocratie que l'exRDA. Il est vrai aussi que ces pays pourraient négocier avec la Commission une période de transition avant de devoir appliquer complètement certaines règles de l'UE. En revanche, il est clair qu'ils devront accepter en principe l'acquis communautaire dans sa totalité et uniformément avant d'être autorisés à rejoindre l'UE et que cet acquis sera mis en application via les procédures supranationales et centralisées habituelles.

On peut imaginer deux scénarios menant à une déstabilisation. Dans le plus plausible de ces scénarios, on verrait - sous l'effet des forces contradictoires exercées d'un côté par les anciennes promesses politiques sur l'élargissement et de l' autre par la rigidité des procédures de négociation de la Commission - des accords d'adhésion contenant des exigences intenables, formulés avec la complicité tacite des représentants de la Commission dans l'espoir qu'une application laxiste de ces exigences serait tolérée. Mais, comme le décalage entre ce qui est légalement requis et ce qui est économiquement et politiquement faisable ne manquera pas d'être flagrant, le déficit d'application des règlements ne pourra pas passer inaperçu. Chez les candidats à l'accession, le cynisme envers la législation européenne ne manquerait pas de saper le respect de la loi en général et, tandis que les entreprises et les groupements d'intérêt des actuels pays de l'UE prendraient conscience des avantages acquis par les nouveaux venus à cause de leur mauvaise application des règlements européens, la légitimité de ces derniers pourrait être remise en question par les « vieux » États membres aussi.

Dans l'autre scénario - moins probable -, la Commission n'admettrait aucun laxisme dans l'application des règles et utiliserait ses importants pouvoirs de sanction pour forcer les gouvernements des pays entrants à s'en tenir à la lettre des accords signés. Dans ce cas-là, des règlements prévus pour des démocraties stables, fonctionnant avec une économie de marché hautement compétitive, seraient appliqués à des pays d'Europe centrale et orientale économiquement arriérés et politiquement fragiles; on pourrait observer les mêmes effets que dans certains pays en voie de développement qui furent forcés par le FMI et la Banque mondiale de réduire drastiquement leur déficit budgétaire et leurs dépenses publiques afin de pouvoir obtenir des prêts internationaux. À la différence de l'Allemagne de l'Est, où les répercussions de

1. Katharina Holzinger, Peter Knöpfel (eds), Environmental policy in a European Union of Variable Geometry? The Challenge of the Next Enlargement, Basel, Helbing \& Lichtenhahn, 2000 ; Katharina Müller, Andreas Ryll, Hans-Jürgen Wagener (eds), Transformation of Social Security: Pensions in Central-Eastern Europe, Heidelberg, Physical Verlag, 1999. 
« l'occidentalisation » forcée furent atténuées non seulement par les transferts financiers massifs en provenance de l'Ouest mais aussi par l'intégration des élites politiques au sein des solides institutions de la République fédérale, l'application rigide des acquis européens en Europe centrale et orientale pourrait délégitimer non seulement l'intégration européenne, mais aussi les régimes démocratiques des nouveaux États membres.

Pour éviter ces deux scénarios peu prometteurs, l'Union doit trouver des moyens légitimes de différencier les règles qui s'appliquent à des États membres dont la situation économique, sociale et politique rendrait l'exécution uniforme de règles européennes uniformes soit impossible soit grosse de risques inacceptables. En l'occurrence, cette différenciation ne pourrait être laissée à la discrétion de la Commission et de la Cour de justice dans les cas individuels, où l'absence de procédures standardisées et générales ainsi que le manque de transparence encourageraient les requêtes spéciales et provoqueraient des soupçons de favoritisme et de corruption. Cependant, la formulation de procédures générales mais différenciées au sein du processus législatif pourrait se révéler extrêmement difficile - et cela pourrait être très contre-productif dans des domaines où les différences les plus marquées entre pays ne peuvent être valablement représentées par des indicateurs quantifiables, et où il ne suffirait pas de répondre à ces divergences en adoptant une législation contenant des exigences quantitatives variables pour des États membres appartenant à des catégories différentes. En dernière analyse, toutefois, ces difficultés ne présentent qu'une différence de degré avec les problèmes rencontrés par l'UE lorsqu'il s'agit de débattre sérieusement avec les actuels États membres des questions que nous allons maintenant aborder.

\section{LA SAUVEGARDE DE L'ÉTAT PROVIDENCE EN EUROPE}

L'intégration européenne a réussi, au-delà de toutes les espérances, son élargissement et son approfondissement du marché intérieur, ainsi que sa création d'une union monétaire. Mais, tandis que ces objectifs économiques sont en train de se réaliser, la capacité des gouvernements nationaux à influencer le cours de leur économie nationale et de modeler leur paysage social a été fortement réduite. Ainsi, l'union monétaire n'a pas seulement privé les États de leur capacité à réagir à des problèmes économiques en réévaluant leur monnaie, mais elle a aussi créé une situation où la politique monétaire européenne - qui doit nécessairement répondre aux conditions qui prévalent dans la zone euro dans son ensemble - ne pourra plus être adaptée aux économies nationales dont les taux d'inflation et de croissance se trouvent au-dessous ou au-dessus des taux européens moyens, ce qui aura pour conséquence de déstabiliser ces économies. Cependant, alors que l'inévitable inadaptation de la politique monétaire de la $\mathrm{BCE}$ oblige à trouver des stratégies compensatrices au niveau national, les gouvernements se voient sévèrement contraints dans leurs décisions de politique fiscale par le Pacte de stabilité, qui punit les pays souffrant d'une croissance lente, mais ne peut rien faire contre les gouvernements dont l'économie est en surchauffe et fortement inflationniste ${ }^{1}$.

1. Henrik Enderlei, «Wirtschaftspolitik in der Währungsunion. Die Auswirkungen der Europäischen Wirtschafts- und Währungsunion auf die Finanz- und Lohnpolitischen Institutionen in den Mitgliedsländern », thèse de doctorat, Cologne, Max-Planck Institute for the Study of Societies, 2001. 
Parallèlement, les politiques de libéralisation et de dérégulation européennes ont éliminé la possibilité d'utiliser les industries du secteur public pour amortir les effets du chômage ; elles ne permettent plus d'utiliser les services publics et la régulation par les services financiers comme des outils de la politique industrielle régionale ou sectorielle, tandis que les règlements européens sur la concurrence interdisent largement le recours aux aides de l'État et aux financements publics dans de tels cas. Dans le même temps, l'intégration européenne a fait disparaître toutes les barrières légales à la libre circulation des biens, des services, du capital et des travailleurs. Les citoyens européens ont le droit de résider où ils veulent sur le territoire des États membres et les traités restreignent très fortement la possibilité pour les États membres de favoriser leurs ressortissants.

En bref, comparé à leur éventail de choix politiques il y a deux ou trois décennies, les gouvernements nationaux ont perdu en grande partie leur capacité à infléchir la croissance et l'emploi de leur pays - à moins que cet infléchissement n'aille dans le sens de davantage de dérégulations et de réductions fiscales, qui sont, elles, parfaitement acceptables dans le cadre réglementaire de l'UE. Dans le même temps, les gouvernements sont fortement incités à user de ces stratégies de l'offre afin d'attirer ou de retenir des entreprises et des investissements qui menacent de se délocaliser dans des zones où les coûts de production sont plus bas et les revenus du capital après impôt, plus élevés. Parallèlement, les syndicats se voient obligés d'accepter des baisses de salaire ou des conditions d'emploi moins avantageuses afin de sauver les emplois existants. À l'opposé, les États providence généreux sont aussi tentés de réduire l'accès à leurs prestations et à leurs services sociaux financés par l'impôt pour éviter l'immigration de bénéficiaires potentiels.

Prises ensemble, ces pressions et ces tentations entrent en conflit avec les engagements et les aspirations politiques des pays qui, dans les décennies qui suivirent la guerre, avaient adopté un large éventail de politiques redistributives et correctrices, créant ainsi « des économies sociales de marché » capables de modérer le mode capitaliste de production par la régulation des conditions de production et d'emploi, et capables de modifier la redistribution inégale des économies capitalistes par des allocations et des services publics financés par l'impôt progressif. Aussi longtemps que les barrières économiques sont restées sous contrôle national, de telles politiques pouvaient être entièrement compatibles avec un développement économique vigoureux, puisque les détenteurs de capitaux n'avaient que le choix d'investir au niveau national, tandis que les entreprises étaient généralement en mesure de répercuter les coûts de la régulation et de la fiscalité sur des consommateurs captifs. Donc, en l'absence de contraintes économiques fortes, la politique jouait son rôle, gouvernements et syndicats étaient, dans une large mesure, libres d'opter pour un État socialement très ou peu généreux et pour un marché du travail fortement ou peu régulé. Mais, avec la disparition des barrières économiques, ces choix économiques sont devenus ou bien un avantage comparatif ou bien un handicap dans la course européenne aux investissements, à la production et à l'emploi.

Si ces pressions et ces tentations ne se sont pas encore vraiment manifestées dans les politiques des États providence européens, c'est en grande partie à cause des résistances politiques face aux réformes que le simple intérêt économique rendraient nécessaires. Mais cette résistance politique se paye souvent d'un taux plus faible de croissance et d'emploi ${ }^{1}$. Les efforts fournis par certains pays en vue d'accroître simultanément la productivité du travail et du capital peuvent aussi retarder l'apparition de

1. Fritz W. Scharpf, Vivien A. Schmidt (eds), Welfare and Work in the Open Economy. Vol. 1, From Vulnerability to Competitiveness. Vol. 2, Diverse Responses to Common Challenges, Oxford, Oxford University Press, 2000. 
ces phénomènes. Dans la mesure où ces efforts sont couronnés de succès, le coût unitaire du travail peut être réduit sans réduction des salaires ou des prestations sociales, et les profits avant impôts peuvent croître suffisamment pour contrecarrer les effets de l'impôt sur les sociétés sur les bénéfices après impôt. Mais si d'autres pays s'engagent dans cette voie, les avantages acquis sont rapidement anéantis et, en tout cas, la croissance accélérée de la productivité n'est généralement pas suivie d'un accroissement de la demande équivalent et elle peut donc conduire à un déclin général des taux d'emploi dans les secteurs industriels et de services qui sont exposés à la compétition internationale ${ }^{1}$.

Il n'est donc pas surprenant que les pays et les groupements d'intérêt qui se sont appuyés sur une économie fortement réglementée et sur des prestations et des services sociaux généreux se tournent désormais vers l'Union européenne pour lui réclamer la protection ou la création d'un « modèle social européen » qui assumeraient les fonctions que les États-nation ne peuvent plus assurer de la même manière depuis l'achèvement du marché intérieur et de l'union monétaire. En théorie, ces demandes sont tout à fait recevables. Avant la mise en place de l'intégration économique européenne, politiques libérales («market-making ») ou dirigistes («market-correcting ») avaient leur place au niveau national ; les lois sur la concurrence n'avaient pas un statut supérieur à celui de la législation gouvernant les services postaux ou l'attribution de subventions aux secteurs ou aux régions défavorisées. De plus, au niveau européen, la très décriée Politique agricole commune a démontré qu'il était possible de trouver un équilibre entre politiques libérales et politiques dirigistes. Alors, pourquoi ne pas combiner aussi les politiques favorisant et protégeant le marché européen des biens, des services et du capital avec celles visant à harmoniser la fiscalité et les règles du marché ?

Économiquement, cela serait évidemment envisageable. Alors qu'il est beaucoup question actuellement des effets déstabilisants de la «mondialisation », il est un fait que l'économie mondiale est beaucoup moins intégrée, et donc beaucoup moins contraignante, que le marché intérieur européen. De plus, l'Union européenne est beaucoup moins dépendante des importations et des exportations que ses États membres et, avec la création de l'union monétaire, elle est devenue beaucoup moins vulnérable face aux vicissitudes des marchés de capitaux internationaux. D'un point de vue purement théorique, donc, une gestion macroéconomique, une politique industrielle, une régulation et une taxation des activités commerciales continuent d'être des options économiquement envisageables pour l'Union européenne. Cela correspondrait en fait à la promesse faite par Jacques Delors d'une « dimension sociale » attachée à la création du marché intérieur d'abord, et de l'union monétaire ensuite. Mais malheureusement, cette promesse n'a pas été tenue et ne pouvait pas l'être dans le cadre institutionnel actuel de l'organisation européenne.

\section{LE DILEMME EUROPÉEN : CONSENSUS ET UNIFORMITÉ}

Comment se fait-il que les problèmes abordés plus haut ne peuvent trouver de solutions satisfaisantes dans le cadre institutionnel et politique de l'Union euro-

1. Fritz W. Scharpf, «Economic Changes, Vulnerabilities, and Institutional Capabilities », dans Fritz W. Scharpf, Vivien A. Schmidt (eds), Welfare and Work in the Open Economy. Vol. 1, From Vulnerability..., op. cit., p. 21-124. 
péenne ? On peut faire un raccourci et répondre que, par le passé, l'Europe a toujours dû satisfaire deux exigences - uniformité et consensus large - et que des solutions efficaces aux problèmes soulevés ici ne pourraient pas satisfaire ces deux exigences à la fois.

Le postulat selon lequel la politique européenne doit prendre la forme de règles uniformes applicables de manière égale par tous les États membres, sous-tend l'idée de marché intégré, ainsi que l'idée d'une législation européenne : un marché parfaitement unifié exige des normes uniformes pour les produits afin de supprimer toute barrière non-douanière, un processus de régulation uniforme pour créer un « terrain de jeu égal » et des règles de concurrence uniformes pour empêcher toute discrimination. Et même si l'on doit, d'un point de vue pragmatique, tolérer des différences au niveau des législations nationales, cette tolérance ne peut pas s'étendre à la législation européenne - dont l'unique raison d'être est justement de surpasser et de supprimer tous les obstacles nationaux au libre échange et de créer un espace légal unifié.

Il y a eu, bien sûr, des compromis inévitables. On a dû octroyer aux candidats à l'adhésion des périodes de grâce avant qu'ils ne puissent appliquer les acquis communautaires dans tous les domaines politiques ; certains États membres n'appartiennent pas encore à l'union monétaire ou à «l'espace Schengen »; il a fallu accepter des clauses d'exemption dans d'autres domaines aussi. Mais on considère que toutes ces exceptions ne peuvent pas invalider l'engagement de chaque État membre à respecter des règles communes. Ainsi, bien que toutes les régions d'Europe ne bénéficient pas de fonds structurels, les règles gouvernant la mise à disposition de ces fonds sont les mêmes partout. En principe, donc, la politique européenne, qu'elle soit adoptée selon le mode supranational, selon des procédures de prise de décision conjointe ou selon le mode intergouvernemental, est censée être uniforme sur tout le territoire des États membres de l'Union.

L'exigence d'une politique fondée sur le consensus s'applique évidemment aux décisions prises selon le mode intergouvernemental et, en pratique, elle s'applique aussi aux procédures conjointes, même si le Conseil décide à la majorité qualifiée. Formellement, elle ne s'applique pas au mode supranational et centralisé. Mais, comme je le faisais remarquer plus haut, la délégation de la prise de décision aux pouvoirs supranationaux est en général sous-tendue par des accords intergouvernementaux préalables sur les objectifs à atteindre. De toute façon, aucun des nouveaux défis qui attendent l'Europe ne pourrait être relevé en suivant la voie supranationale et hiérarchique dans le cadre institutionnel actuel. Au contraire, il faudrait en passer soit par la voie intergouvernementale, soit suivre la procédure de prise de décision conjointe, qui toutes deux s'appuient bien évidemment sur un large consensus.

Dans le domaine de la sécurité européenne, une politique uniforme serait hautement désirable du simple point de vue de l'efficacité. Mais, pour les raisons mentionnées plus haut, il est fort peu probable qu'on puisse dégager un consensus permettant d'envisager un déploiement militaire efficace et rapide, dans un domaine encombré par les considérations de souveraineté nationale et les problèmes de positionnement face à l'engagement militaire - divergences héritées du passé ou inscrites dans des orientations politiques normatives. Inversement, il semble que, concernant l'élargissement à l'Est, seule une politique uniforme puisse trouver l'accord de tous les. États membres actuels, ce qui éliminerait la nécessité d'une adaptation des « acquis communautaires » aux conditions socio-économiques radicalement différentes des pays de l'Europe centrale et orientale. S'il existe des solutions, il faudrait les trouver à la lumière des problèmes de politique sociale rencontrés actuellement par les États 
membres après l'intégration économique. Ce sont ces questions que je traiterai en priorité.

La doctrine du consensus et de l'uniformité a fonctionné correctement quand il s'agissait de réaliser les objectifs de base de l'intégration économique, elle a fonctionné aussi dans le contexte politico-économique des années 1980 où la marche vers la libéralisation des marchés et l'harmonisation des normes des produits avait, en principe du moins, le soutien des consommateurs et des producteurs des États membres. Le principe du consensus ne fonctionne plus aussi bien lorsqu'on touche à la régulation des processus de production. Comme les réglementations concernant la protection de l'environnement, la sécurité des travailleurs et les conditions d'emploi sont susceptibles de faire augmenter les coûts de production et les prix, elles ne reçoivent généralement pas le soutien des organisations de consommateurs ; par ailleurs, si les producteurs des pays où règne une réglementation forte sont prêts à soutenir une harmonisation à hauts niveaux de protection afin d'assurer un « terrain de jeu égal », ce n'est pas le cas dans les pays ayant un faible niveau de réglementation. Par conséquent, les régulations européennes des processus de production se feront le plus souvent a minima, afin de pouvoir être acceptées par tous les pays. Mais, par comparaison, il reste plus facile d'obtenir un consensus sur la régulation des processus de production que dans les domaines de la politique sociale et de l'harmonisation fiscale.

L'une des raisons en est que la compétition fiscale et réglementaire n'affecte pas nécessairement tous les États membres. Par exemple, les petits pays peuvent en réalité voir leurs revenus augmenter en attirant les capitaux mobiles par de faibles taux d'imposition, tandis que les grands pays ne pourraient pas suivre le même mouvement sans voir leurs revenus fortement entamés '. Cependant, les différences dans le développement économique jouent un rôle encore plus déterminant. Ainsi, le coût des dépenses d'aide sociale et des services sociaux publics considérés comme normaux dans les pays scandinaves ne pourrait tout simplement pas être supporté par les États les moins riches tels que la Grèce, l'Espagne ou le Portugal, sans parler des pays de 1'Est candidats à la future adhésion. S'il n'y avait que ce problème toutefois, et si l'on pouvait définir l'uniformité en termes relatifs, on pourrait encore s'entendre sur des normes reflétant les différences dans la capacité à payer des États membres se trouvant à des étapes différentes du développement économique. Mais, bien que la GrandeBretagne et la Suède soient aussi riches, il n'est pas sûr qu'elles tombent d'accord sur une politique européenne commune concernant le social ou les relations patronat-syndicats.

C'est ainsi parce que les États providence en sont venus à définir des lignes de partage très divergentes entre les fonctions dévolues à l'État et celles laissées à la gestion privée, soit des familles soit du marché ${ }^{2}$. Tous les pays fournissent une aide sociale aux nécessiteux, mais en Scandinavie et en Europe continentale, l'État offre aussi une assurance sociale indexée sur le salaire qui est censée assurer le niveau de vie d'une famille à moyens revenus en cas de chômage, de maladie, de handicap et au moment de la retraite. En Grande-Bretagne et dans d'autres pays anglo-saxons, en revanche, les travailleurs ayant un revenu moyen ou supérieur ont appris à investir dans des fonds privés pour faire face à ces éventualités. D'autre part, seuls les États

1. Vivek H. Dehedjia, Philipp Genschel, «Tax Competition in the European Union», Politics and Society, 27, 1999, p. 403-430.

2. Gosta Esping-Andersen, The Three Worlds of Welfare Capitalism, Princeton, Princeton University Press, 1990 ; Fritz W. Scharpf, Vivien A. Schmidt, op. cit. 
providence scandinaves offrent des prestations sociales universelles et de haute qualité qui permettent aux femmes mariées et aux mères de se libérer de leurs tâches familiales, tout en fournissant au secteur public des emplois qui ont élevé le taux de féminisation du marché du travail à des niveaux record. Dans les pays anglo-saxons, continentaux et d'Europe du Sud, en revanche, ces prestations relèvent de l'univers familial ou du marché. On rencontre, entre les États membres de l'Europe, des différences tout aussi marquantes dans le domaine des relations patronat-syndicats '.

Ces différences structurelles ne sont pas simplement de nature technique, elles ont aussi une grande signification politique. Elles correspondent à des aspirations sociales fondamentalement divergentes qui sont, en gros, l'héritage historique des doctrines sociales liées aux partis politiques « libéraux », "Chrétiens-démocrates » et « sociaux-démocrates » ${ }^{2}$. Mais le point peut-être le plus important est le suivant : les citoyens de tous les pays se sont habitués à bâtir des projets de vie en tablant sur la pérennité des systèmes existants de protection sociale et de taxation, et toute tentative de les remplacer par des systèmes européens de moins bonne qualité susciterait de farouches réactions d'opposition. Les électeurs britanniques ne pourraient tout simplement pas supporter le niveau d'imposition qui permet au généreux système social suédois de survivre; les familles suédoises ne pourraient pas vivre avec le faible niveau de services sociaux et éducatifs qui prévaut en Allemagne; tandis que les médecins allemands et leurs patients s'uniraient pour protester contre un système à la britannique. Bref, il n'y a pas de «modèle social européen » unique pouvant donner lieu à une harmonisation ${ }^{3}$.

Ainsi, les gouvernements nationaux, ayant des comptes à rendre à leurs électeurs, ne sont d'aucune façon en mesure de tomber d'accord sur des solutions européennes concernant les fonctions clé de l'État providence. Cela n'empêche pas l'adoption de normes minimales sur les droits sociaux et des travailleurs, soit par le biais de directives émanant du Conseil européen, soit au travers d'accords émanant du « dialogue social » entre chefs des organisations syndicales et patronales. Mais, comme ces normes doivent être acceptables pour tous les États membres, elles ne doivent pas seulement être économiquement viables pour les pays les moins riches, mais aussi compatibles avec le type de relations patronat-syndicats et les systèmes sociaux déjà en place. Il n'est pas surprenant, dans ces conditions, que seules des réglementations très peu exigeantes aient pu franchir ce double obstacle ${ }^{4}$, ce qui signifie aussi que, même si ces réglementations participent à l'élévation du niveau de protection sociale dans les pays anglo-saxons et du sud, elles ne contribueront guère à soulager les pressions concurrentielles s'exerçant sur les systèmes sociaux plus développés des pays scandinaves et de l'Europe continentale.

1. Colin Crouch, Industrial Relations and European State Traditions, Oxford, Clarendon Press, 1993 ; Bernhard Ebbinghaus, Jelle Visser, Trade Unions in Western Europe since 1945, Londres, Macmillan, 2000.

2. G. Esping-Andersen, op. cit.

3. Maurizio Ferrera, Anton C. Hemerijck, Martin Rhodes, The Future of Social Europe: Recasting Work and Welfare in the New Economy, Oeiras, Celta, 2000.

4. Wolfgang Streeck, «Neo-Voluntarism: A New European Social Policy Regime ? », European Law Journal, 1, 1995, p. 31-59; Wolfgang Streeck, «Industrial Citizenship under Regime Competition: The Case of European Works Councils », Journal of European Public Policy, 4, 1997, p. 643-664. 


\section{SUBSIDIARITÉ OU UNIFORMITÉ IMPOSÉE ?}

Si l'exigence de consensus au centre de la politique décisionnelle européenne empêche de trouver des solutions efficaces, il semble possible d'envisager deux réponses institutionnelles extrêmes : l'une d'elles consisterait à passer outre l'européanisation et à laisser les États membres régler les problèmes eux-mêmes ; l'autre réponse consisterait à mettre en œuvre des réformes institutionnelles permettant d'imposer des solutions européennes en dépit des oppositions qu'elle pourraient soulever. La contribution allemande à l'actuel débat constitutionnel tente de combiner, sous des formes diverses, ces deux idées. Cependant, dans le cas des problèmes qui nous intéressent ici, aucune de ces deux solutions radicales ne paraît appropriée.

\section{LA SUBSIDIARITÉ}

Le principe de «subsidiarité », tel que défini dans l'article 5, II du TCE, met en avant deux conditions : l'action européenne est permise seulement s'il est vrai que « les objectifs de l'action envisagée ne peuvent être réalisés de manière suffisante par les États membres » et si, d'autre part, il est vrai que ces objectifs peuvent « être mieux réalisés au niveau communautaire ». Le principe de subsidiarité ne propose toutefois aucune solution dans le cas où la première condition est vraie et la seconde fausse ce qui est précisément le cas des problèmes qui nous occupent.

Pour deux d'entre eux, il est pourtant clair que l'européanisation est la seule solution possible : en dépit de tous les problèmes posés par la mise en place d'une Politique étrangère et de sécurité commune, il ne fait aucun doute que ses objectifs ne pourraient être atteints par des actions autonomes au niveau national. De même, si, dans le cadre du processus d'élargissement à l'Est, il est nécessaire de définir des règles permettant un ajustement des acquis communautaires, il est impossible que l'Union laisse aux États candidats le soin de définir ces règles. C'est donc seulement dans la défense de l'État providence européen que le principe de subsidiarité peut être considéré comme une solution possible. Pour les auteurs « libertariens », la subsidiarité apparaît comme une solution formellement préférable parce qu'elle assure la supériorité constitutionnelle des libertés économiques garanties par les traités sur les politiques sociales adoptées au niveau national ${ }^{1}$. Suivant une argumentation formelle différente, la subsidiarité est aussi défendue par Giandomenico Majone ${ }^{2}$ qui considère la Communauté européenne comme un État régulateur dont les règles sont légitimées par des critères d'efficacité. Comme les politiques sociales de redistribution ne sauraient se voir légitimées de la sorte, il s'ensuit qu'elles doivent rester sujettes aux choix politiques effectués par des gouvernements nationaux démocratiquement élus.

Dans une perspective plus positive que normative, l'impact de la mondialisation économique sur les politiques d'aide sociale nationales fait l'objet d'une vaste contro-

1. Ernst-Joachim Mestmäcker, «Zur Wirtschaftsverfassung in der Europäischen Union », dans Rolf H. Hasse, Josef Molsberger, Christian Watrin (eds), Ordnung in Freiheit : Festgabe für Hans Willgerodt zum 70. Geburtstag, Stuttgart, Fischer, 1994, p. 263-292.

2. Giandomenico Majone, Regulating Europe, Londres, Routledge, 1994. 
verse dans la littérature théorique et empirique ${ }^{1}$. On peut raisonnablement conclure que ces études tendent généralement à souligner la résistance (conservatrice) des États providence face aux pressions de la concurrence économique. De plus, des études de cas comparatives ont montré qu'il existait des exemples d'adaptations politiques et d'ajustements créatifs ayant permis à certains pays de maintenir ou d'atteindre un haut niveau de compétitivité et d'emploi sans sacrifier leurs aspirations sociales ${ }^{2}$. À la lumière de ces enseignements, il est aussi suggéré aux partis de centre-gauche et aux syndicats qu'au lieu de perdre leur temps inutilement en cherchant à s'européaniser, ils feraient mieux de créer des structures de «solidarité compétitive » au niveau national.

Il faut noter, cependant, que les pays en question bénéficiaient dès le départ de conditions économiques et institutionnelles favorables et qu'il existe en fait davantage de pays qui sont soit aux prises avec des difficultés économiques, soit dans l'obligation d'opérer des coupes claires dans leurs prestations et leurs services sociaux entrấnant une importante augmentation des inégalités sociales. De plus, la plupart des études citées envisagent les effets à long terme de la «mondialisation », plutôt que l'impact récent de l'achèvement du marché intérieur et de l'union monétaire. Quoi qu'il en soit, les solutions purement nationales au sein de l'Union européenne seront toujours contraintes par l'asymétrie constitutionnelle existant entre le système légal européen et les systèmes légaux nationaux. Tant qu'il ne sera pas possible de renouveler complètement la boîte à outils des politiques économiques et sociales utilisées dans l'après-guerre par les gouvernements nationaux, les options politiques qui resteront ouvertes au niveau national seront souvent trop restreintes pour permettre de trouver des solutions efficaces.

Ces réserves s'appliquent aussi aux demandes faites par le gouvernement français et par les Länder allemands de réviser les traités afin de poser des limites explicites aux compétences européennes et ce, dans le but de protéger les services et les infrastructures publics, ainsi que les politiques industrielles des gouvernements nationaux et subnationaux ${ }^{3}$. Dans l'optique qui est ici la nôtre, les conséquences de telles demandes sont au mieux ambivalentes. Même si ces mesures pouvaient être adoptées

1. Stefan Sinn, «The Taming of Leviathan : Competition among Governments », Constitutional Political Economy, 3, 1993, p. 177-221 ; Vito Tanzi, Taxation in an Integrating World, Washington, Brookings, 1995 ; Geoffrey Garret, Partisan Politics in the Global Economy, Cambridge, Cambridge University Press, 1998 ; Duane Swank, «Funding the Welfare State : Globalization and the Taxation of Business in Advanced Market Economies », Political Studies, 46, 1998, p. 671-692 ; Fritz W. Scharpf, Vivien A. Schmidt, op. cit. ; Paul Pierson (ed.), The New Politics of the Welfare State, Oxford, Oxford University Press, 2001 ; Evelyne Huber, John D. Stephens, Development and Crisis of the Welfare State: Parties and Policies in Global Markets, Chicago, University of Chicago Press, 2001.

2. Cf. Anton C. Hemerijck, Martin Schludi, «Sequences of Policy Failures and Effective Policy Responses », dans Fritz W. Scharpf, Vivien A. Schmidt (eds), op. cit., p. 125-128. ; Fritz W. Scharpf, « Economic Changes... », art. cité ; Evelyne Huber, John D. Stephens, op. cit. Des différences semblables ont été relevées relativement à l'impact des politiques de libéralisation européennes sur la réglementation nationale du secteur des services publics (Adrienne Héritier, Dieter Kerwer, Christoph Knill, Dirk Lehmkuhl, Michael Teutsch, Anne-Cécile Douillet, Differential Europe: European Union Impact on National Policy-Making, Boulder, Rowman \& Littlefield, 2001).

3. Antoine Lyon-Caen, Véronique Champeil-Desplats (dir.), Services publics et droits fondamentaux dans la construction européenne, Paris, Dalloz, 2001 ; Gerhard Schroeder, « SPD Leitantrag... », cité ; CDU/CSU, «Vorschlag für einen Europäischen Verfassungsvertrag », $2001<$ http://www.welt.de/daten/2001/11/271127de298494.htx >. 
contre l'avis de la Grande-Bretagne, des Pays-Bas ou d'autres pays ayant réussi leur libéralisation, il est difficile de voir comment elles pourraient desserrer les contraintes légales du marché intérieur qui, après tout, constituent le cœur des acquis européens. En tout état de cause, ces mesures ne pourraient sans doute pas imposer de restrictions sur la libre circulation des biens, des services et des capitaux et donc, ne pourraient pas préserver leurs politiques régulatrices du marché des pressions économiques engendrées par la concurrence fiscale et réglementaire. Mais, dans le même temps, ces tentatives pour limiter les compétences de l'Europe pourraient fort bien réussir à dresser des barrières légales venant annihiler les efforts encore timides en vue d'établir ou de renforcer les pouvoirs européens dans des domaines tels que l'environnement, le social et l'emploi, où justement les solutions nationales ne sont plus suffisantes. En d'autres termes, l'autonomie nationale ne peut plus assurer le succès des politiques régulatrices du marché. Ce dont on a besoin, au contraire, c'est de solutions européennes qui (comme pour la PAC) viennent compléter l'intégration économique par l'européanisation des fonctions régulatrices du marché.

\section{SOLUTIONS IMPOSÉES}

Si ces solutions européennes sont bloquées par absence de consensus, il pourrait alors sembler opportun d'envisager des réformes institutionnelles réduisant les possibilités de veto. Après tout, c'est de cette manière que l'Acte unique européen a permis la réussite du projet de marché intérieur, en introduisant le vote à la majorité qualifiée au sein du Conseil. C'est cette même idée qu'inclut implicitement la vision d'un État fédéral européen défendue par Joschka Fischer ' - État dans lequel une majorité simple aux deux chambres législatives suffirait à définir les choix politiques européens. Sur un plan moins politique et technocratique, ce sont des espoirs similaires qui ont inspiré l'appel, apparemment plus modéré, à une « revitalisation de la méthode communautaire » - appel émis par la Commission dans son « Livre blanc sur un gouvernement européen » (COM 2001/428). Selon la Commission, cette revitalisation impliquerait les changements suivants dans les pratiques de gouvernement :

- Le Conseil et le Parlement devraient limiter leur implication dans la législation européenne primaire à la définition des «éléments essentiels (droits et obligations fondamentaux, conditions de leur mise en œuvre), en laissant à l'exécutif le soin de veiller aux modalités techniques par 1'application de règles de droit dérivé » (p. 25).

- Pour déterminer le contenu de ces règles dérivées, la Commission devrait être autonome, guidée, certes, par le Conseil et le Parlement, mais non plus gênée dans son action par les Comités de régulation et de gestion (p. 36).

- Afin d'accélérer le processus législatif, le Conseil « devrait voter dès qu'une majorité qualifiée semble se dessiner, plutôt que de poursuivre les discussions dans la recherche de l'unanimité » (p. 27).

- De plus, afin de protéger son monopole d'initiative législative contre les effets de la recherche d'un consensus, « la Commission s'est engagée à retirer ses propositions lorsque la négociation interinstitutionnelle sape [...] les objectifs desdites propositions » (p. 27).

\section{Joschka Fischer, art. cité.}


Prises toutes ensemble, ces propositions réduiraient le nombre de questions devant être réglées par des négociations intergouvernementales ou interinstitutionnelles, permettant ainsi que des questions «non-essentielles » soient réglées unilatéralement par la Commission. Il ne fait aucun doute que de telles règles auraient parfaitement fonctionné pour l'harmonisation des normes de produits dans le cadre de l'Acte unique européen de 1986, où l'intérêt commun l'emportait sur les intérêts nationaux. Mais qu'adviendrait-il dans le cas où des solutions nationales déjà en place étaient menacées? Prenons le cas des systèmes de retraite, où les «modalités techniques » pourraient avoir un impact important sur l'espérance de vie des individus et donc, faire l'objet de luttes acharnées entre groupements d'intérêt et partis politiques, soulevant ainsi de violentes protestations susceptibles de mettre en danger un gouvernement. Dans ces conditions, la diversité nationale ne peut pas être considérée comme étant illégitime, elle fait partie intégrante du processus de légitimation des croyances et des pratiques qui sous-tend l'organisation politique européenne à plusieurs niveaux. Chaque fois que ces conditions se présentent, toute tentative de passer outre la diversité légitime en imposant des règles européennes uniformes par la «méthode communautaire » et le vote majoritaire au Conseil risque de faire éclater l'Union.

La Commission ne semble pas être consciente des limites inhérentes au vote majoritaire dans l'UE. En affirmant qu'il « est temps de reconnaître que l'Union est passée d'un processus diplomatique à un processus démocratique » (p. 35), la Commission semble penser que l'UE pourrait fonctionner comme une démocratie majoritaire dans laquelle les positions minoritaires pourraient être ignorées. Mais, l'Union n'est pas (encore) une organisation démocratique unifiée et ses électeurs ne constituent pas (encore) un « corps politique » intégré, prenant part à des débats publics et à des batailles de partis au niveau européen, et possédant des organes pleinement représentatifs et efficaces. Au contraire, l'Europe continue à dépendre de la légitimité démocratique de ses États membres '. Le vote à la majorité qualifiée est devenu un outil pratique quand il s'agit d'accélérer les décisions du Conseil dans des domaines où la divergence dans les préférences politiques n'est pas politiquement sensible au niveau de chaque État. Mais lorsque tel n'est pas le cas, les gouvernements des États membres ont de très bonnes raisons pratiques et théoriques de prendre le temps de trouver des solutions consensuelles. Pour l'heure en tout cas, la politique européenne doit être consensuelle si elle veut être légitime.

Étant donné la diversité des situations économiques, des cultures politiques, des structures institutionnelles, des héritages politiques et des opinions publiques dans les États membres, il paraît inévitable que de nombreux choix se situant sous le seuil des «principes essentiels » aient un fort impact politique et soient totalement inacceptables dans un pays ou un autre. Pour l'instant, ces écueils sont contournés par la recherche de solutions consensuelles évitant les incompatibilités avec des contraintes nationales spécifiques grâce aux négociations intergouvernementales complexes qui ont lieu lors de la phase préparatoire aux décisions du Conseil, ainsi que durant la phase de mise en œuvre. Les propositions de la Commission viendraient court-circuiter les mécanismes de recherche d'un consensus dans le cadre du mode conjoint de

1. M. Rainer Lepsius, «The European Union as a Sovereignty Association of a Special Nature », dans Christian Joerges, Yves Mény, J. H. H. Weiler (eds), op. cit., p. 213-222; J. H. H. Weiler, «Fischer: The Dark Side», dans Christian Joerges, Yves Mény, J. H. H. Weiler (eds), op. cit., p. 235-247. 
prise de décision, sans pour autant être en mesure de créer une nouvelle base de légitimation.

Le Livre blanc a, bien sûr, raison de souligner que les résultats des procédures consensuelles appliquées dans les modes de prise de décision intergouvernementaux et conjoints laissent beaucoup à désirer si l'on s'en tient à des critères d'efficacité. Les processus décisionnels sont malaisés et lents, et leurs résultats, lorsqu'on parvient à un accord, en restent souvent au niveau du plus petit dénominateur commun, ou bien se caractérisent par un excès de détails contraignants et de paperasserie. Il est facile, dans ces conditions, de souscrire au désir de la Commission de se libérer elle-même, et de libérer la politique européenne de ce carcan « procédural ». Mais, dans les domaines politiques sensibles où les divergences nationales sont légitimes, la solution proposée par le Livre blanc, qui, essentiellement, remplacerait les procédures consensuelles par des votes à la majorité et par le pouvoir de décision unilatéral de la Commission, ne pourrait pas être imposée aux gouvernements des États membres et ne serait pas formellement acceptable de toute façon. Si l'Union européenne veut pouvoir faire face aux problèmes posés par la diversité légitime, elle ne peut le faire en imposant des solutions uniformes. Il faut, au contraire, instaurer des modes de gouvernement qui puissent s'accommoder de la diversité tout en traitant avec efficacité les problèmes qui ne peuvent être résolus qu'au niveau européen.

\section{COMMENT RELEVER LE DÉFI DE LA DIVERSITÉ LÉGITIME}

Si la diversité des situations économiques, des institutions et des spécificités nationales est un enjeu politique tel qu'il n'est pas possible de trouver un accord sur des politiques européennes uniformes et si les politiques strictement nationales ne peuvent répondre à un problème urgent, on peut logiquement imaginer deux types de solutions : des pouvoirs européens centralisés qui réduiront les spécificités politiques sensibles au niveau national et des politiques européennes différenciées capables de prendre en compte les divergences et les spécificités nationales.

\section{RÉDUCTION DE L'IMPACT POLITIQUE}

La logique de la première option trouve une illustration dans le domaine des normes de produits, où il a été extrêmement difficile de trouver un régime commun d'autorisation de mise sur le marché des produits pharmaceutiques via une « reconnaissance mutuelle » ou via une harmonisation des procédures d'autorisation. En revanche, la mise en place d'une Agence centralisée européenne et de procédures centralisées pour concéder des autorisations d'expérimentation de nouveaux médicaments à l'échelle européenne s'est révélée remarquablement consensuelle et efficace '. Cela semble pouvoir s'expliquer par le fait qu'il ne s'agissait que d'une disposition complémentaire ne nécessitant aucun changement dans les organisations et les procédures nationales existantes et qui ne menaçait pas les spécificités nationales

1. Jürgen Feick, « The Efficacy of European Public Policy Solutions : The Case of Market Entry Regulations for Pharmaceuticals », Cologne, Max-Planck Institute for the Study of Societies, 2001. 
(par exemple, cas des médicaments homéopathiques). De même, l'allocation de fonds européens pour le développement économique prête moins à la controverse que les tentatives de coordination des programmes nationaux pour l'aide au développement ; inversement, les efforts actuels en vue de coordonner les programmes de recherche et de développement nationaux au sein d'un «Espace de recherche européen » auront plus de mal à aboutir que les accords passés sur les Programmes cadres européens.

La question est de savoir si le même genre de solutions pourrait s'appliquer utilement aux problèmes dont nous traitons ici, par exemple, à la création d'une Force de sécurité européenne commune. Apparemment, les efforts actuels pour renforcer les institutions communes de planification et de prise de décision sont prometteurs ${ }^{1}$. Mais, d'une manière générale, il semble peu probable que les difficultés actuelles puissent être entièrement aplanies aussi longtemps qu'elles tourneront autour du contrôle européen sur le déploiement des forces nationales. Les questions de souveraineté et de spécificités nationales auraient-elles le même impact politique si l'objet des décisions européennes n'était plus l'affectation de contingents de forces armées nationales à une intervention commune, mais la création d'une force de réaction rapide, recrutée sur la base d'une armée européenne de volontaires et financée entièrement sur le budget européen (et peut-être sur les revenus d'une taxe européenne sur les intérêts du capital) ? Bien sûr, une telle force européenne serait petite comparée à la somme totale des armées nationales, mais son équipement, ses méthodes d'entraînement, son commandement et sa logistique devraient agir en étroite collaboration avec les forces armées des États membres de l'Union et avec l'OTAN. Sa taille et son potentiel technique pourraient être suffisants pour effectuer de brèves missions de maintien ou de rétablissement de la paix sans avoir à dépendre de l'engagement actif de forces nationales - dont l'existence comme forces de réserve donnerait néanmoins plus de poids et de crédibilité à ces missions.

Si cette force d'intervention authentiquement européenne venait à voir le jour, les gouvernements nationaux devraient toutefois assumer la responsabilité politique de chacun de ses mandats. Mais on peut espérer qu'un accord sur ces mandats ne rencontre pas trop de réticences au niveau national. En même temps, leur mise en œuvre pourrait être dans une plus large mesure déléguée à une structure de commandement supranationale. Je ne peux pas savoir si une telle solution trouverait un soutien politique. Tout ce que je peux dire, c'est que les éléments sur lesquels elle repose semblent acceptables au regard de ce que nous savons des procédures décisionnelles dans le cadre institutionnel de l'organisation politique européenne.

\section{UNE COOPÉRATION RENFORCÉE ?}

Cependant, concernant les autres problèmes examinés ici, le recours à des pouvoirs centralisés ne serait guère approprié. Il faudrait, au contraire, faire appel à des formes «d'intégration différenciée » capables de prendre en compte des conditions et des spécificités nationales divergentes. Une solution pourrait être fournie par (une version révisée) des dispositions sur une « coopération renforcée » figurant au Titre VII du traité sur l'Union européenne. Conformément à l'article 44 du TUE, des groupes

1. Jolyon Howorth, «European Defence and the Changing Politics of the European Union... », art. cité. 
d'États membres pourraient recourir à l'éventail complet des décisions de la CE, adoptées et mises en ouvre via les procédures habituelles de la CE - à l'exception du fait que le vote au Conseil est restreint aux États membres participants.

En ayant recours à ces options, les pays à forts taux de prélèvement pourraient harmoniser le taux de leurs impôts sur les bénéfices ; les pays fortement industrialisés pourraient adopter conjointement des règles environnementales plus contraignantes que les États membres moins développés; et les États du Sud et de l'Est pourraient se mettre d'accord sur des normes s'appliquant aux systèmes d'aide au revenu de base en fonction des ressources. De même, les pays finançant leur système de santé par un système d'assurances obligatoires pourraient harmoniser les réglementations régissant l'autorisation et la rémunération des prestataires de service ; les pays envisageant la privatisation partielle de leurs systèmes de retraite publics pourraient harmoniser les réglementations concernant les options de placements ; les pays souhaitant conserver des transports publics efficaces et bon marché pourraient conjointement réguler la concurrence entre prestataires publics et privés ; et les pays souhaitant conserver une télévision de service public pourraient en faire de même.

Le principal avantage d'une telle coopération renforcée serait, bien sûr, que des groupes de pays partageant les mêmes situations économiques ou institutionnelles, ou les mêmes choix politiques, pourraient s'entendre sur des solutions communes impossibles à mettre sur pied à l'échelle de l'Europe tout entière. Mais surtout, les règles ainsi adoptées auraient le même poids que les lois européennes, sur l'axe vertical aussi bien qu'horizontal. Verticalement, elles auraient un effet contraignant sur les décideurs politiques nationaux et sub-nationaux, avec pour conséquence de supprimer toute tentation pour les membres des différents groupes de recourir à la compétition fiscale et réglementaire. Savoir si de tels accords seraient économiquement intéressants ou bien, au contraire, contre-productifs est une question que les gouvernements devraient examiner à la lumière du fait que la compétition économique a tendance à être plus forte entre États de même niveau de développement. Toutefois, c'est d'abord l'effet horizontal qui devrait être pris en compte dans de nombreux cas. Comme les règlements ainsi adoptés auraient le même poids que la loi européenne, ils se trouveraient au même niveau constitutionnel que toutes les autres règles de la législation européenne. À cet égard, l'équilibre entre politiques favorisant le marché et politiques régulant le marché, qui caractérise la Politique agricole commune, pourrait s'appliquer aux politiques de santé et de transports publics.

Alors, pourquoi employons-nous le mode conditionnel ? Parce que, dans le traité d'Amsterdam, les conditions d'un renforcement de la coopération ont été si étroitement définies qu'on n'en trouve actuellement aucun exemple '. D'une part, la coopération renforcée est autorisée seulement pour des groupes comportant « au moins une majorité d'États membres » (art. 43 (1, d) TUE). D'autre part, selon l'article 11 (2, para 1) du TCE, la décision d'autoriser la coopération renforcée dépend d'un vote du Conseil « statuant à la majorité qualifiée sur proposition de la Commission » et, selon l'article 11 (2, para 2) du TCE, cette décision peut être empêchée par l'opposition d'un seul État membre invoquant « des raisons de politique nationale importantes et qu'il

1. Il serait plus exact de dire que les exemples qui, en fait, existent bel et bien ne tombent pas sous le coup des règles gouvernant «la coopération renforcée ». L'union monétaire est devenue le plus important de ces exemples, mais «l'espace Schengen », même après avoir été soumis au Traité, n'inclut pas non plus tous les membres de l'Union, et il en va de même pour la PECSD. 
expose ». Ces conditions extrêmement restrictives seraient rendues légèrement moins draconiennes si le traité de Nice était ratifié, mais l'exigence d'avoir au moins huit États membres pour constituer un groupe rendrait caduque pratiquement tous les exemples donnés plus haut. De plus, le traité d'Amsterdam a exclu tous les accords qui « affecteraient l'acquis communautaire et les mesures prises au titre des autres dispositions desdits traités » (art. $43(1, \mathrm{e})$ TUE) ou qui constitueraient « une entrave aux échanges entre les États membres » et qui provoqueraient « une distorsion des conditions de la concurrence entre ceux-ci » (art. $11(1$, e) TCE).

Pourquoi une telle hostilité à l'égard de la coopération renforcée ? L'une des réponses se trouve certainement dans la défense farouche de l'acquis communautaire par la Commission ainsi que dans la prédominance des discours sur l'intégration économique et sur la libéralisation au sein de la Commission. Mais pourquoi est-ce que les gouvernements - qui pourraient passer outre la Commission lors du processus de réforme des traités - partagent cette opinion ? Cela est lié, à mon avis, à un problème de formulation. Sans même tenir compte des différents termes qui ont été employés depuis les années 1970 - «vitesse variable », "géométrie variable », «cercles concentriques », « deux niveaux », «noyau » ou, plus récemment, «groupe pionnier » - la notion d'intégration différenciée a été généralement associée à l'idée d'un progrès dans un espace à une dimension, un axe unique allant de moins à plus d'intégration '. Il s'agissait de permettre à une « avant-garde » d'États membres, qui en étaient capables et désireux, d'aller de l'avant - ce qui semblait signifier que les autres pays allaient se retrouver dans une arrière-garde, avec le risque de se voir relégués dans une sorte de seconde classe de l'Europe. Étant donné les décennies d'incompréhension et d'appréhension qui pèsent sur ce débat, il n'est pas vraiment surprenant que les règles extrêmement restrictives adoptées à Amsterdam aient été à peine libéralisées au sommet de Nice.

La façon dont est formulé ce problème est doublement malencontreuse : d'une part, parce qu'elle empêche une innovation institutionnelle utile, d'autre part, parce qu'elle empêche aussi que soit reconnu le fait que l'européanisation préservatrice des aspirations à réguler le marché des États providence européens ne peut aboutir que si l'on permet à l'intégration d'avancer dans un espace à plusieurs dimensions. Cela n'implique pas simplement que les différences de développement économique devraient jouer un rôle dans la définition et la mise en œuvre de l'acquis communautaire. Cela signifie aussi qu'il est faux de considérer le « modèle social européen », qui fait partie de l'agenda européen depuis le sommet de Lisbonne, comme constituant un objectif unique. Ainsi, cela n'a aucun sens de considérer, soit le modèle scandinave, soit le modèle anglo-saxon, comme l'avant-garde que les autres pays devraient rattraper. Au contraire, l'européanisation des politiques sociales devrait permettre aux deux groupes de pays de maintenir et de développer leurs propres solutions en fonction de leur vision de la solidarité sociale; elle devrait permettre aux États providence bismarckiens de rechercher des solutions communes à leurs problèmes communs; et elle devrait aider les États candidats à l'accession à mettre en œuvre des institutions

1. Claus-Dieter Ehlermann, « Differentiation, Flexibility, Closer Cooperation : The New Provisions of the Amsterdam Treaty », European Law Journal, 4 (3), 1998, p. 246-270 ; Claus Giering, Europa Zwischen Zweckverband und Superstaat : die Entwicklung der politikwissenschaftlichen Integrationstheorie im Prozess der europäischen Integration, Bonn, Europe-UnionVerlag, 1997; Neil Walker, "Sovereignty and Differential Integration in the European Union », European Law Journal, 4 (4), 1998, p. 355-388 ; Gráinne de Búrca, Joanne Scott (eds), Constitutional Change in the EU: From Uniformity to Flexibility ?, Oxford, Hart, 2000. 
de protection sociale économiquement et politiquement viables, sans qu'ils soient obligés d'adopter un plan européen uniforme '. Les mêmes réflexions s'appliquent à la politique de l'environnement ${ }^{2}$. Tout en s'appliquant seulement à des sous-groupes (variables) d'États membres, la coopération renforcée conserverait toutes les caractéristiques de la législation européenne. Ainsi, la Commission continuerait à initier les lois, le rôle législatif du Parlement (ou de sous-groupes au Parlement) serait maintenu, les États membres pourraient être mis en minorité au Conseil, et les directives et règlements adoptés seraient interprétés et exécutés au titre de la législation européenne par la Commission et la Cour de justice. En d'autres termes, par le biais d'une diversification maîtrisée, la coopération renforcée favoriserait l'européanisation de la politique publique et, de surcrô̂t, empêcherait sa re-nationalisation. C'est pour cette raison même, cependant, que la coopération pourrait se révéler une solution trop rigide dans des domaines où il serait prématuré de passer de l'autonomie nationale à la coordination via 1'application rapide et stricte de lois européennes.

\section{MÉTHODE OUVERTE DE COÖRDINATION ?}

On peut remédier à ces rigidités par la « méthode ouverte de coordination » qui avant la lettre - fut définie par le traité de Maastricht pour la coordination des politiques économiques des États membres (art. 98 et 99 TCE) et appliquée aux politiques de l'emploi dans le traité d'Amsterdam (art. 125 à 130 TCE), avant que le sommet de Lisbonne $n$ 'introduise cette appellation générique et l'applique à un certain nombre d'objectifs de politique industrielle et sociale. Cette méthode suppose que les gouvernements des États membres, tout en se mettant d'accord sur certains objectifs ou problèmes de politique qu'ils considèrent comme des questions « d'intérêt commun », conservent, au final, la responsabilité de leurs choix politiques. L'essentiel de cette méthode consiste en une procédure itérative qui commence par un rapport de la Commission au Conseil européen - rapport suivi des directives émanant du Conseil, ellesmêmes fondées sur une proposition de la Commission. En réponse à ces directives, les gouvernements des États membres présentent «un plan d'action national » annuel et un rapport sur les mesures prises - qui est ensuite évalué à la lumière de « points de référence » comparatifs par la Commission et un comité permanent de hauts fonctionnaires. Ces évaluations viennent à leur tour nourrir les rapports et directives annuels suivants, mais elles peuvent aussi conduire à l'adoption de recommandations spécifiques du Conseil adressées individuellement aux États membres. Cependant, « l'harmonisation des dispositions législatives et réglementaires des États membres » est explicitement exclue des mesures que le Conseil peut adopter (art. $129 \mathrm{TCE}$ ).

Il est trop tôt pour mesurer l'efficacité de cette méthode ouverte de coordination, mais son potentiel et ses limites apparaissent assez clairement. Comme les États membres conservent la maîtrise de leurs choix politiques, ils restent aussi en mesure de répondre à la spécificité de leur situation économique, institutionnelle et politique. Néanmoins, la méthode ouverte de coordination implique que les gouvernements nationaux se concentrent sur des problèmes ou des objectifs définis conjointement et

1. Katherina Müller, The Political Economy of Pension Reform in Central-Eastern Europe, Cheltenham, Edward Elgar, 1999; Katharina Müller, Andrea Ryll, Hans-Jürgen Wagener (eds), op. cit.

2. Katharina Holzinger, Peter Knöpfel (eds), op. cit. 
qu'ils envisagent leur propre choix politique en fonction du problème donné. De plus, en soumettant leurs propres performances à une évaluation comparative, à l'examen de leurs pairs et au regard du public, la coordination ouverte pourrait offrir des conditions favorables à « un apprentissage par monitoring » ${ }^{1}$ et elle pourrait aussi détourner les gouvernements de leurs stratégies protectionnistes qui seraient contre-productives si elles étaient adoptées par tous.

Cependant, il est clair que tous ces effets bénéfiques dépendent essentiellement de la volonté des acteurs nationaux de s'engager dans des processus de coordination européens qui, en définitive, n'ont pas force d'obligation. Si cette volonté existe, alors les recommandations européennes peuvent jouer le rôle de puissants arguments dans les discussions de politique nationale; sinon, les plans d'action nationaux ne feront que refléter les vieilles habitudes; et les pauvres officiers de liaison assistant aux innombrables réunions de Bruxelles devront assumer la responsabilité de politiques nationales sur lesquelles ils n'ont aucune influence. Mais, même si l'apprentissage politique fonctionne au niveau national, il est un fait que les politiques ainsi adoptées - bien qu'elles puissent améliorer la qualité des stratégies nationales d'ajustement ne peuvent créer des lois européennes et donc n'aideront en rien à réduire l'impact du marché intérieur et de l'union monétaire dans les différents domaines de la politique nationale et elles ne permettront pas d'alléger les contraintes légales et économiques qui pèsent sur les responsables politiques.

\section{DIRECTIVES CADRES ET COORDINATION OUVERTE?}

Dans son Livre blanc sur la gouvernance européenne, la Commission vante les mérites de la méthode ouverte de coordination, cependant elle rappelle que « cette méthode ne doit pas être utilisée quand une action législative relevant de la méthode communautaire est possible » (p. 26). Pourtant, c'est peut-être ce qui permettrait à la coordination ouverte d'être plus efficace. Imaginons que le Conseil et le Parlement acceptent de prendre en compte la recommandation du Livre blanc de réduire la législation aux «éléments essentiels », mais que - au lieu de déléguer la formulation de règlements spécifiques à la Commission et à des comités - la mise en œuvre de ces « éléments essentiels » soit laissée aux mains des gouvernements ; en l'état, cela correspondrait au modèle des « directives cadres », qui, selon le Livre blanc, devait être utilisé plus souvent (p. 24). Il se peut qu'il ne le soit pas plus souvent actuellement en raison de la méfiance mutuelle de gouvernements qui doutent de la volonté de leurs partenaires d'appliquer des mandats législatifs contraignants ou de résister aux tentations protectionnistes.

Mais que se passerait-il si des directives cadres étaient associées à la méthode ouverte de coordination? Les États membres devraient alors décrire les mesures qu'ils ont prises et faire un rapport sur leurs effets, tandis que leurs performances respectives seraient comparées par la Commission et évaluées par leurs pairs et par le Conseil. Si cette évaluation devait révéler des problèmes d'ordre général, la législation-cadre pourrait être amendée et renforcée. Concernant less déficits d'exécution spécifiques à

1. Charles F. Sabel, «Learning by Monitoring: The Institutions of Economic Development », dans Neil Smelser, Richard Swedberg (eds), Handbook of Economic Sociology, Princeton, Princeton University Press, 1995, p. 137-165 ; Jelle Visser, Anton C. Hemerijck, Learning Ahead of Failure : A Research Agenda, Ms University of Amsterdam, 2001. 
certains pays, le Conseil pourrait par ailleurs prendre des décisions légalement contraignantes ou bien autoriser la Commission à engager les poursuites habituelles pour infraction au droit communautaire. En d'autres termes, les États membres continueraient à jouir d'une grande liberté dans la définition du contenu et de la procédure des directives cadres, afin que ces dernières soient adaptées à la situation et aux spécificités nationales. Toutefois, s'ils devaient abuser de cette liberté, aux yeux de leurs pairs siégeant au Conseil, on pourrait toujours avoir recours à des sanctions centralisées et à des procédures de mise en œuvre, au titre de « force dissuasive ».

Comme le contrôle supplémentaire induit par la coordination ouverte pourrait rendre les directives cadres plus acceptables aux yeux de la Commission et aux yeux des États se méfiant les uns des autres, l'efficacité de la coordination ouverte serait d'autant plus grande une fois que ces procédures seraient incluses dans le contexte de directives cadres qui, comme toutes les autres directives, ont force de loi européenne. Ainsi, sur l'axe vertical, leur mise en œuvre n'est pas laissée à la discrétion des gouvernements et les acteurs responsables des choix de politique nationale dans les différents domaines ne pourraient se permettre de simplement passer outre les accords pris au niveau européen. De même, les obligations légales concrètes aideraient à surmonter l'opposition intérieure aux choix politiques requis pour appliquer les règlements de bonne foi. Mais, ce qui est capital dans le contexte actuel, c'est que, sur l'axe horizontal, les politiques ainsi adoptées auraient, elles aussi, le statut de lois européennes et donc, ne se verraient pas limitées de manière asymétrique par les politiques libérales adoptées au même niveau constitutionnel.

La différenciation pourrait être poussée d'un cran supplémentaire si les directives cadres et la coordination ouverte pouvaient s'appliquer dans le contexte de la « coopération renforcée » dont nous avons parlé plus haut. Dans ce cas-là, on conserverait l'avantage de directives légalement contraignantes, mais celles-ci seraient mieux ciblées sur les problèmes ou les objectifs spécifiques à certains groupes d'États membres, ce qui, par ailleurs, contribuerait largement à un apprentissage politique mutuel.

La politique publique européenne a atteint ses objectifs économiques : intégration du marché intérieur et création de l'union monétaire. Mais elle est à présent confrontée à des problèmes cruciaux qui ne peuvent trouver de solutions dans le cadre actuel des modes de gouvernement de l'Union. Tout en étant différents les uns des autres, ces modes de gouvernement doivent répondre à deux exigences communes : pour être efficace, la politique européenne doit s'appuyer sur un large consensus et doit, au moins en principe, donner naissance à des règles s'appliquant uniformément dans toute l'Union. Ces exigences ont pu, en gros, être satisfaites simultanément au cours des processus décisionnels qui ont mené à l'intégration économique. Tel n'est plus le cas aujourd'hui, alors que l'Europe doit faire face à de nouveaux défis : possibilité d'intervenir rapidement pour maintenir ou rétablir la paix au cours de conflits susceptibles d'affecter la sécurité européenne commune ; nécessité de favoriser le développement économique, social et politique des candidats à l'adhésion en Europe centrale et orientale ; nécessité de préserver la pluralité des «modèles sociaux européens » face aux contraintes et aux pressions des marchés intégrés. J'ai essayé de montrer que, dans chacun de ces domaines, les deux conditions énoncées plus haut - large consensus et réglementation uniforme - ne pouvaient être satisfaites en même temps. 
La cause de cette impossibilité se trouve dans le rôle crucial joué par ce que j'ai appelé la «diversité légitime ». Une fois acquis l'engagement dans le processus d'intégration économique, la défense de la diversité nationale a été pervertie par le recours à un protectionnisme illégitime. Inversement, les initiatives visant à relever ces nouveaux défis se heurtent à des conflits politiques qui trouvent leur origine dans des différences fondamentales au plan économique et institutionnel. Par ailleurs, l'héritage historique, la sensibilité politique de l'électorat constituent un autre obstacle important. Dans des cadres institutionnels nécessitant un large consensus, les gouvernements des États membres ne pourraient pas s'entendre sur des solutions européennes uniformes qui saperaient leur propre légitimité démocratique.

J'ai aussi essayé de montrer que deux manières apparemment évidentes de trancher ce dilemme ne sont en réalité pas viables. Le fait de passer de modes de gouvernement fondés sur le consensus à des prises de décision à la majorité au Conseil et au parlement, ou à la Commission, nous ferait sortir du cadre de la légitimité européenne. L'union n'est pas, et n'est pas près d'être, une démocratie majoritaire, et la Commission ne constitue pas un gouvernement de l'Europe démocratiquement élu. Le vote à la majorité qualifiée au Conseil et la délégation des pouvoirs à la Commission constituent des outils acceptables dans des domaines où les différences nationales n'ont pas un trop fort impact politique. Dans le cas contraire, les gouvernements refuseraient, à juste titre, d'abdiquer leur responsabilité face à des choix qui, au bout du compte, remettraient en cause leur propre légitimité.

Mais si on ne peut légitimement imposer de solutions uniformes aux États «dissidents », l'autre terme de l'alternative consistant à renoncer complètement à l'européanisation en invoquant le principe de « subsidiarité » ne pourrait pas résoudre le dilemme non plus car cela ne permettrait pas de répondre efficacement aux problèmes soulevés ici. Dans le domaine de la Politique étrangère et de sécurité, il est clair que, en l'absence d'européanisation, les gouvernements échoueraient probablement à s'entendre, à moins d'y être forcés par le leadership de la puissance hégémonique américaine. En ce qui concerne l'élargissement à 1'Est, il est clair aussi que, quand bien même l'application uniforme de l'acquis communautaire serait synonyme de désastre, on ne peut laisser la définition de cet acquis entièrement aux mains de chacun des nouveaux adhérents.

Concernant l'État providence, enfin, il est vrai que certains pays ont parfaitement réussi à s'adapter à la mondialisation de l'économie sans abandonner leurs engagements en faveur de la protection sociale. Mais d'autres ont dû choisir entre leur compétitivité et leurs aspirations sociales, tandis que d'autres encore ont échoué sur les deux fronts '. Quoi qu'il en soit, l'engagement héroïque de certains États à maintenir une «solidarité compétitive » au niveau national ${ }^{2}$ est probablement un combat perdu d'avance si ces États doivent non seulement faire face à la mondialisation des marchés mais aussi aux contraintes du libéralisme dogmatique instituées dans le cadre de lois européennes sur la concurrence. Ainsi, les États providence scandinaves sont peut-être économiquement viables. Mais il n'est pas certain qu'ils pourraient survivre si leurs services sociaux publics devaient être libéralisés et devaient affronter la libre concur-

1. Fritz W. Scharpf, Vivien A. Schmidt, op. cit.

2. Wolfgang Streeck, «Competitive Solidarity », dans Karl Hinrichs, Herbert Kitschelt, Helmut Wiesenthal (eds), Kontingenz und Krise. Institutionenpolitik in kapitalistischen und postsozialistischen Gesellschaften. Claus Offe zu seinem 60. Geburtstag, Frankfort, Campus, 2000 , p. 245-262. 
rence de prestataires privés. En bref, si le problème de l'État providence en Europe devait être résolu par le principe de subsidiarité, la décentralisation ne pourrait pas s'appliquer uniquement aux politiques visant à réguler le marché, mais aussi à celles visant à le favoriser, ce qui me paraît fort peu probable.

En d'autres termes, la sortie apparemment facile du dilemme - règle de la majorité ou subsidiarité - se ferait, soit aux dépens de la légitimité, soit aux dépens de l'efficacité. Dans le domaine politiquement sensible de la sécurité européenne, les divergences entre pays pourraient être aplanies s'il ne s'agit pas de déployer des forces nationales sur le terrain, mais de constituer une force armée européenne totalement centralisée. En général toutefois, il sera difficile de trouver des solutions efficaces aussi longtemps qu'on jugera nécessaire que l'européanisation des politiques publiques prenne la forme de règles uniformes applicables à tous les États de l'Union.

Ainsi, si l'on veut que le débat constitutionnel actuel soit utile, il doit tourner autour de nouveaux modes de gouvernement susceptibles de fournir des réponses européennes efficaces aux nouveaux défis qui attendent l'UE et capables de faire une place à la légitime diversité nationale. À la fin de cet article, $\mathrm{j}$ 'ai examiné deux options possibles, « la coopération renforcée » et « la méthode ouverte de coordination », qui sont déjà en germe dans les traités actuels. J'ai souligné le fait que l'immense potentiel de la coopération renforcée ne pourrait pas être exploité avant que les restrictions concernant son adoption ne soient allégées au-delà de ce qui a été défini dans le traité de Nice. La méthode ouverte de coordination pourrait, dans sa forme actuelle, favoriser l'apprentissage politique au niveau national, mais serait incapable d'avoir l'efficacité politique et juridique de solutions européennes. Il serait donc peut-être intéressant d'envisager de combiner «coordination ouverte » et «directives cadres», ces dernières étant juridiquement contraignantes mais laissant aux gouvernements nationaux le soin de déterminer le détail de leur contenu et de leurs modalités d'application.

\section{Traduction Bruno Poncharal}

Fritz W. Scharpf, après avoir étudié le droit et la science politique en Allemagne et à Yale, est devenu, en 1968, professeur de science politique à l'université de Constance. De 1973 à 1984, il est directeur de l'Institut international de management du Centre de recherche de Berlin et, en 1986, il devient directeur de l'Institut Max Planck pour l'étude des sociétés, à Cologne. Il est l'auteur de Crisis and Choice in European Social Democracy, Ithaca, Cornell University Press, 1991 ; Games Real Actors Play : Actor-Centered Institutionalism in Policy Research, Boulder, Westview, 1997 ; Governing in Europe : Effective and Democratic?, Oxford, Oxford University Press, 1999, et (avec Vivien A. Schmidt) Work and Welfare in the Open Economy, Oxford, Oxford University Press, 2000, 2 vol. Ses recherches portent sur la Loi constitutionnelle américaine, la théorie de la démocratie, les politiques publiques dans les bureaucraties ministérielles, le fédéralisme allemand, les études comparées en économie politique et l'intégration européenne. (<scharpf@mpi-fg-koeln.mpg.de >). 


\section{RÉSUMÉ/ABSTRACT}

\section{LA DIVERSITÉ LÉGITIME : NOUVEAU DÉFI DE L'INTÉGRATION EUROPÉENNE}

Discuter à propos d'une constitution européenne nécessite de se confronter à d'importants défis politiques qui ne se rencontrent pas réellement dans le cadre institutionnel actuel de l'Union européenne. Le présent article présente tout d'abord trois modes de gouvernement communautaire distincts : les négociations intergouvernementales, la centralisation supranationale et les décisions conjointes. Dans ces trois modes de gouvernement, l'action européenne appliquée et légitime dépend d'un haut degré de consensus entre les membres des gouvernements des États. $L^{\prime}$ 'article examine ensuite les conditions des réponses européennes aux trois nouveaux défis politiques : l'élargissement à l'Est, la politique communautaire extérieure et de sécurité ainsi que la protection des États providence européens face à l'impact de l'intégration économique et de la libéralisation du marché. Dans chaque cas, on peut voir que les conflits d'intérêt significatifs entre les membres des gouvernements empêchent toute action dans le cadre institutionnel actuel. Prétendant que ces conflits ne peuvent pas être résolus par une évolution vers des institutions à régime majoritaire, l'article conclut en proposant différentes réponses politiques qui permettraient l'action communautaire dans le cadre d'une diversité légitime.

\section{LEGITIMATE DIVERSITY : THE NEW CHALLENGE OF EUROPEAN INTEGRATION}

Discussions about a European constitution ought to focus on substantive policy challenges that cannot be met effectively within the present institutional framework of the European Union. The paper begins by discussing the characteristics of three distinct modes of European policy making - intergovernmental agreement, supranational centralization and joint-decision making. In all three modes, effective and legitimate European action depends on high levels of substantive consensus among member state governments. The paper goes on to examine the requirements of effective European responses to three novel policy challenges - Eastern enlargement, common foreign and security policy and the protection of European welfare states against the impact of economic integration and liberalization. In each case, it can be shown that politically salient conflicts of interest among member governments will prevent effective action within the present institutional framework. Arguing that these conflicts could not be legitimately settled by a move toward majoritarian institutions, the paper concludes with a discussion of differentiated policy responses that would allow European action in the face of legitimate diversity. 